\title{
EFFICIENT OPEN WORLD REASONING FOR PLANNING
}

\author{
TAMARA BABAIAN ${ }^{a}$ AND JAMES G. SCHMOLZE ${ }^{b}$ \\ ${ }^{a}$ Department of Computer Information Systems, Bentley College, Waltham, MA 02452 USA \\ e-mail address: tbabaian@bentley.edu \\ ${ }^{b}$ Department of Computer Science, Tufts University, Medford, MA 02155 USA
}

\begin{abstract}
We consider the problem of reasoning and planning with incomplete knowledge and deterministic actions. We introduce a knowledge representation scheme called PSIPLAN that can effectively represent incompleteness of an agent's knowledge while allowing for sound, complete and tractable entailment in domains where the set of all objects is either unknown or infinite. We present a procedure for state update resulting from taking an action in PSIPLAN that is correct, complete and has only polynomial complexity. State update is performed without considering the set of all possible worlds corresponding to the knowledge state. As a result, planning with PSIPLAN is done without direct manipulation of possible worlds. PSIPLAN representation underlies the PSIPOP planning algorithm that handles quantified goals with or without exceptions that no other domain independent planner has been shown to achieve. PSIPLAN has been implemented in Common Lisp and used in an application on planning in a collaborative interface.
\end{abstract}

\section{INTRODUCTION}

Much progress has been made in the area of planning with a correct but incomplete description of the world, i.e. an open world. However, so far there have been relatively few formalisms proposed for efficient open world representation and reasoning in the domains with a very large or unknown set of individual objects. This paper describes a representation, called PSIPLAN, for planning with incomplete information about the initial state, that handles open world planning problems that have not been shown to be solved by any other implemented domain independent planning system.

For an example of an open world problem, consider a robot operating in a warehouse that is given a goal of delivering box A to the front door. The robot can pick up only those boxes that do not contain fragile items. To pick up box A the robot does not need to know the location of all fragile items, nor the precise contents of box A. It is sufficient to know that A has no fragile items. Thus, in such situations, if the robot knows that (a) no containers have fragile goods except for $B$, it can safely pick up box A. When the list of all containers that the robot might eventually have to reason about is unknown, it is impossible to represent (a) by enumerating all containers that do not have fragile goods.

2000 ACM Subject Classification: I.2.4; I.2.8; F 4.1; F.2.2.

Key words and phrases: Knowledge representation and reasoning, planning with incomplete information.

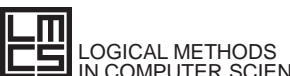

IN COMPUTER SCIENCE
DOI:10.2168/LMCS-2 (3:5) 2006
(C) T. Babaian and J. G. Schmolze (c) Creative Commons 
Thus to include (a) in the robot's description of the state, or to represent a goal such as there is nothing at the front door except for box A, a quantified statement is necessary.

The robot must be able to update quickly and correctly its knowledge of the state that results from performing an action. For instance, after a new container $\mathrm{C}$, whose contents are unknown, is added to the warehouse, the robot's updated state must reflect that (b) there are no containers with fragile goods except for $B$ and possibly $C$.

Effective and efficient operation in a partially known environment may require the ability to satisfy knowledge goals, such as for example, (c) identify all containers with fragile goods that will be shipped to Boston, and sensing actions that return information about the world, such as examining the contents of a box, or determining a box's destination from its label. A robot that is able to reason in a sound and complete manner about its knowledge and lack of knowledge is better equipped to handle such knowledge goals. For instance, knowing only (b) plus that C's destination is Chicago, when given a knowledge goal (c), the robot must be able to conclude that the only necessary information that is missing is the destination of B. Such precision of reasoning ensures that sensing is non-redundant and relies on sound and complete reasoning in the underlying representation.

As this example demonstrates, a representation used in an open-world application must distinguish between propositions whose truth value the agent knows and those whose truth value it does not know. Merely listing all facts marked with their truth value is inefficient when the set of all domain objects is very large because, typically, the number of atoms whose value is known to be false is large. For example, the set of all objects that are not in box A could be very large. Furthermore, such enumeration of known atoms is impossible when the set of all domain objects is infinite or unknown. A key requirement of an open world planning representation with partially unknown or infinite domains is to allow quantification for (at least) negative information while retaining efficient reasoning.

In this paper we present a representation for open world reasoning that uses a class of quantified sentences with exceptions that we call $\psi$-forms. $\psi$-forms represent quantified negative information such as the following statement: there are no containers with fragile goods except for possibly $B$ and $C$. We present an efficient calculus for $\psi$-forms, that includes a sound and complete entailment procedure that takes polynomial time under certain reasonable assumptions on the structure of $\psi$-forms.

Further, we present a formalism called PSIPLAN, based on $\psi$-forms, for reasoning and planning in partially known worlds. PSIPLAN offers a unique combination of tractability, expressivity and completeness of reasoning. This makes it uniquely suited for applications where the set of all domain objects can never be acquired, and the ability to generate and explore alternative plans while avoiding redundant execution is critical. One of the advantages of PSIPLAN compared to other representation used in open world planners, is the fact that all reasoning about actions and states in PSIPLAN is carried out without an explicit manipulation of the set of possible worlds. This property reduces a planner's sensitivity to the amount of irrelevant information, which causes some planners that use explicit enumeration of the possible worlds to blow up.

A partial order planning algorithm (31]) called PSIPOP based on PSIPLAN is presented in [5]. PSIPOP is sound and complete for the planning domains in which the set of all domain objects is infinite or can never be fully acquired. As a partial order planner, PSIPOP produces a solution in a form of a partially ordered set of steps, which can be linearlized or used as a basis for a parallelized plan. The goal language of PSIPOP includes 
quantified goals with exceptions such as (b) that no other implemented domain independent planner has been shown to handle.

An extension of PSIPLAN that includes sensing actions and knowledge goals is presented in 2]. A planner that uses the extended representation for planning with sensing and interleaved execution (PSIPOP-SE) was used in a collaborative interface called Writer's Aid 3]. Writer's Aid helps an author writing an academic manuscript by simultaneously and autonomously identifying, locating and downloading relevant bibliographic records and papers from the author's preferred local and Internet sources. The use of PSIPLAN at the core of Writer's Aid ensures the expressiveness of its goal language, and its ability to precisely identify the missing information and never engage in redundant search, while exploring all possible courses of action.

In this paper we make the following contributions:

- We present a complete description of the language of $\psi$-forms and $\psi$-form calculus, and report on the complexity of the calculus operations. The $\psi$-form calculus is an integral and critical part of PSIPLAN reasoning, and, thus, its algorithms, complexity and completeness properties bear direct effect on the properties of PSIPLANbased planners (5, 2, 3], as well as a Graphplan-style (9]) conformant planner currently under development.)

- We introduce PSIPLAN representation of an agent's incomplete state of knowledge and illustrate it with examples. We further present PSIPLAN's action language and a procedure for state update after an action, and prove important properties of the update procedure, including its completeness.

1.1. Prior Approaches. The lack of universally quantified reasoning in open-world planners (e.g. 11, 17, 14, 36, [40, 13], 1], 25], 32, ) precludes their use in domains in which the set of all objects is not known, very large, or infinite ([39, [16]).

On the other hand, situation calculus-based approaches (e.g. [18, 34, 26]) have the expressivity of full first order logic (FOL), and thus admit planning problems with arbitrary quantified formulas. However, a complete planner based on the unrestricted situation calculus, i.e. that relies upon the full FOL, is impossible due to the undecidability of entailment in FOL. Recently, Liu and Levesque have presented a subset of situation calculus, with a tractable, sound and complete action projection under certain restrictions (30]). Other related approaches to reasoning about actions incorporating first-order features are presented in 37] and 35. These works are reviewed in Section [5.

The LCW (for Locally Closed Worlds) language of Etzioni et. al. [16] is designed to achieve expressivity and tractability for open world reasoning and is most closely related to PSIPLAN. LCW sentences specify the parts of the world for which the agent has complete information. It does this by collecting formulas $\Phi$ where the agent knows the truth value of every ground instance of $\Phi$. For example, if the agent knows all fragile items that are in box $B$, it states $L C W(\operatorname{In}(x, B) \wedge$ Fragile $(x))$. Combined with a propositional database that states that In(Vase, B), Fragile(Vase), the agent can conclude that nothing is fragile in $B$ except for the Vase.

LCW reasoning, although tractable, is incomplete 16, 15. There are two sources of incompleteness in LCW: (1) incompleteness of inference and (2) inability of LCW statements to represent exceptions, i.e., the inability to state that the agent knows the value of all instantiations of formula $\Phi$ except some. These are the key difference between the LCW 
and PSIPLAN representations; PSIPLAN reasoning is complete, and PSIPLAN can also express what exactly is not known via the $\psi$-form exceptions mechanism. Adding a similar mechanism to the LCW framework would require the development of new entailment procedures, methods for state update and operations underlying the planning techniques akin to those developed in this paper.

As a result of the lack of exceptions in LCW sentences, when one or more instances of $\Phi$ is unknown, $L C W(\Phi)$ cannot be stated. This limitation on the expressive power will sometimes cause known information to be discarded from the LCW knowledge base upon updating it after an action, even when the effects of the action are completely specified, do not cause information loss, and create no new objects. For example, consider the result of moving an object $C u p$ from some other box to box $B$ in the situation where $\operatorname{LCW}(\operatorname{In}(x, B) \wedge$ Fragile $(x))$ is asserted. If it is not known whether the Cup object is fragile, the LCW statement above no longer holds and thus must be discarded, effectively discarding from the knowledge base all instances of $\operatorname{In}(x, B) \wedge$ Fragile $(x)$ that are known to be false.

PSIPLAN subsumes a large part of the LCW language. Every knowledge state represented by an LCW-based representation can also be represented in PSIPLAN, except for those situations which require an LCW statement $L C W(\Phi)$, where $\Phi$ contains atoms that unify when all variables are renamed to be distinct ${ }^{1}$. On the other hand, there are states of knowledge that PSIPLAN can represent accurately, but LCW cannot.

Both LCW and PSIPLAN representations can be used in planning with sensing [2, 3]. Since LCW's reasoning is incomplete, however, planners based on LCW are inherently incomplete. To help remedy this, LCW based planners use sensing actions to find out facts they cannot infer, but this only works when an appropriate sensing action is both available and not too costly. In general, there is no effective replacement for sound and complete reasoning.

The LCW [16] representation is extended in [28, 19] to handling exceptions. However, both of these works only consider the setting in which there are no actions that can change the world, do not address a changing world or planning, and do not present any methods that would make these extensions amenable to their use in reasoning about actions.

\subsection{A brief look at PSIPLAN.}

Example 1.1. A robot operating in a warehouse is told that there are no fragile goods in any of the boxes except possibly for the box marked FragileStuff, i.e.

$$
\forall g, c . \neg B o x(c) \vee \neg \operatorname{In}(g, c) \vee \neg \text { Fragile }(g) \vee c=\text { FragileStuff }
$$

Here $\operatorname{Box}(c)$ states that $c$ is a box, $\operatorname{In}(g, c)$ states that item $g$ is in container $c$, and Fragile $(g)$ states that $g$ is fragile. Note that (1.1) does not state whether or not FragileStuff actually contains any fragile items.

In PSIPLAN, statement (1.1) is represented by the following $\psi$-form that represents a conjunction of all ground clauses ${ }^{2}$ that can be obtained by instantiating the formula $\neg \operatorname{Box}(c) \vee \neg \operatorname{In}(g, c) \vee \neg$ Fragile $(g)$ in all possible ways except for instantiations in which $c=$ FragileStuff. This is written as the $\psi$-form

$$
\psi=[\neg \operatorname{Box}(c) \vee \neg \operatorname{In}(g, c) \vee \neg \text { Fragile }(g) \text { except }\{\{c=\text { FragileStuff }\}\}] .
$$

\footnotetext{
${ }^{1}$ These limitations are related to the definition of fixed length $\psi$-forms presented in Section 2 and are critical to the tractability of $\psi$-form reasoning.

${ }^{2} \mathrm{~A}$ clause is a disjunction of literals. A clause is ground if it contains no variables.
} 
whose interpretation is exactly the same as (1.1). Here and below, lowercase letters in $\psi$-forms denote implicitly universally quantified variables, while symbols that start with a capital letter denote constants.

Suppose, it is also known that a bottle of wine is the only fragile item. Consequently, it is in the FragileStuff box, i.e.

$$
\text { Box(FragileStuff), In(Wine, FragileStuff), Fragile(Wine) }
$$

With PSIPLAN, the original situation comprised by (1.1) and (1.3) is represented as the following state of knowledge

$$
s=\left\{\begin{array}{l}
\psi=[\neg \operatorname{Box}(c) \vee \neg \operatorname{In}(g, c) \vee \neg \text { Fragile }(g) \text { except }\{\{c=\text { FragileStuff }\}\}], \\
\text { Box }(\text { FragileStuff }), \text { In }(\text { Wine, FragileStuff }), \text { Fragile }(\text { Wine })
\end{array}\right\}
$$

Now suppose that a new container Box10 is brought into the room whose contents are completely unknown. In the resulting situation, the location of all fragile goods is known except for those that might be in Box10. The PSIPLAN state update would yield the following new state of knowledge $s^{\prime}$ by adding the atom Box(Box10) to $s$ and adding an exception to $\psi$ yielding

$$
\psi^{\prime}=[\neg \operatorname{Box}(c) \vee \neg \operatorname{In}(g, c) \vee \neg \text { Fragile }(g) \text { except }\{\{c=\text { FragileStuff }\},\{c=\text { Box } 10\}\}] .
$$

The updated state of knowledge $s^{\prime}$ represents the new situation precisely:

$$
\begin{aligned}
& s^{\prime}= \\
& \left\{\begin{array}{l}
\psi^{\prime}=[\neg B \text { Box }(c) \vee \neg \operatorname{In}(g, c) \vee \neg \text { Fragile }(g) \text { except }\{\{c=\text { FragileStuff }\},\{c=\text { Box } 10\}\}], \\
\text { Box }(\text { FragileStuff }), \text { Box }(\text { Box } 10), \text { In }(\text { Wine, FragileStuff }), \text { Fragile }(\text { Wine })
\end{array}\right\}
\end{aligned}
$$

PSIPLAN's action language includes actions with $\psi$-form preconditions. For example, the action lift of lifting object $B$, requires that there be no fragile items in it, i.e.

$$
[\neg \operatorname{In}(g, B) \vee \neg \text { Fragile }(g)]
$$

When an agent whose state of knowledge is described with $s^{\prime} \cup \operatorname{Box}(B o x 5)$ is given a goal of lifting box Box5 from its location, it will establish that the precondition $[\neg \operatorname{In}(g, B o x 5) \vee \neg$ Fragile $(g)]$ of the lift action is entailed by $\psi^{\prime}$ and proposition Box $(B o x 5)$. Indeed, if no boxes contain fragile goods, except for the box FragileStuff and possibly Box10, then there are no fragile goods in Box5. The PSIPLAN reasoning algorithms that are involved in this inference do not expand the universal quantification in the universal base and do not require the knowledge of all domain objects by the agent.

Another illustration of the advantage of PSIPLAN's ability to reason with quantified sentences is an example from the blocks world domain. PSIPLAN eliminates the need for predicate $C l e a r(B)$ as a way of stating that nothing is on block $B$. Instead, PSIPLAN's representation uses $[\neg O n(b, B)]$. The advantage of using the latter representation is that the fact that block $A$ is on block $B$ by itself implies that block $B$ is not clear, eliminating the need to state $\neg C$ lear $(B)$ as another effect of moving block $A$ onto $B$.

This use of quantified preconditions distinguishes PSIPLAN from other representations (11], 7], 14, [36], 40, [13, 11, 25], 32]), that only admit actions with preconditions limited to atoms or literals. On the other hand, many of these conformant planners handle actions with conditional effects, which are absent from PSIPLAN. While PSIPLAN can be easily extended to represent actions with conditional effects, complete planning with conditional effects is in the complexity class $\Sigma_{2} P$ (e.g. [6] 38]), while complete planning 
with PSIPLAN appears to be an NP-complete problem. This issue is further discussed in Section 5.2 .

PSIPLAN admits procedures for entailment and state update after an action (including actions that introduce a new object) that are sound, complete and take polynomial time. In particular, the complexity of entailment grows linearly with respect to the number of $\psi$-forms in the knowledge base when the number of literals, variables and exceptions in each $\psi$-form are bounded. The complexity bound on $\psi$-form reasoning is polynomial in the number of exceptions.

The rest of the paper is organized as follows: Section 2 formally defines $\psi$-forms and presents a few simple properties. In Section 3. we present the $\psi$-form calculus and complexity results. Section 4 introduces the PSIPLAN representation of a state of knowledge, actions and state update after an action. Section [ 5 contains an overview of the related work. Finally, Section [6] summarizes and draws conclusions.

\section{The language of $\psi$-Forms}

2.1. Definitions and notation. We assume no function symbols except for constants in the language. The number of constants is infinite.

The general form of a $\psi$-form is:

$$
\psi=\left[\neg Q_{1}\left(\overrightarrow{x_{1}}\right) \vee \ldots \vee \neg Q_{k}\left(\overrightarrow{x_{k}}\right) \text { except }\left\{\sigma_{1}, \ldots, \sigma_{n}\right\}\right]
$$

$k \geq 1$ and $n \geq 0$, and each $Q_{i}\left(\overrightarrow{x_{i}}\right)$ is any atom whose only variables are $\overrightarrow{x_{i}}$. The clause $\neg Q_{1}\left(\overrightarrow{x_{1}}\right) \vee \ldots \vee \neg Q_{k}\left(\overrightarrow{x_{k}}\right)$ is called the main clause of $\psi$ and is denoted by $\mathcal{M}(\psi)$. The set of all variables of the main clause, i.e. the set $\vec{x}=\bigcup_{i=1}^{k} \vec{x}_{i}$, is denoted by $\mathcal{V}(\psi)$.

Each $\sigma_{i}$ is a substitution on a non-empty subset of variables in $\mathcal{V}(\psi)$, that binds a variable to another variable from $\mathcal{V}(\psi)$, or to a constant. The set of all substitutions appearing in a $\psi$-form is denoted $\Sigma(\psi)$. Each $\sigma_{i}$ represents exceptions of $\psi$. Thus, a $\psi$-form can be abbreviated as $[\mathcal{M}(\psi)$ except $\Sigma(\psi)]$.

When $\Sigma(\psi)$ is empty, we call such a $\psi$-form simple and write $[\mathcal{M}(\psi)]$ instead of $[\mathcal{M}(\psi)$ except \{\}$]$. A simple $\psi$-form with no variables is called a singleton and represents a single ground clause.

Given a $\psi$-form $\psi=[\mathcal{M}(\psi)$ except $\Sigma(\psi)]$ we will need to refer to the following:

- a simple $\psi$-form $[\mathcal{M}(\psi)]$ that is obtained from the main clause of $\psi$, called main part.

- simple $\psi$-forms that are obtained by instantiating the main clause with substitutions from $\Sigma(\psi)$, called exception forms. For each $\sigma_{i}$ from $\Sigma(\psi)$, we call $\mathcal{M}(\psi) \sigma_{i}$ the i-th exception clause denoted $\mathcal{E}_{i}(\psi) . \mathcal{E}(\psi)$ is the set of all of $\psi$ 's exception forms: $\left\{\left[\mathcal{E}_{i}(\psi)\right] \mid 1 \leq i \leq\|\Sigma(\psi)\|\right\}$.

We use $C$ and $V$ to denote respectively the maximum number of literals and number of variables in the main clause for a given set of $\psi$-forms. We use $E$ to denote the maximum size of the set $\Sigma(\psi)$. The cardinality of each predicate is assumed to be constant bounded, thus the time for unification of two literals is also constant bounded.

Unless noted otherwise, everywhere in this paper symbols $x, y, z, x_{1}, y_{1}, z_{1}, \ldots$ denote variables, capital letters $A, B, \ldots$ denote constants, and $\psi, \psi_{1}, \ldots$ - denote $\psi$-forms. Also, we assume that the variables in two distinct $\psi$-forms are always renamed to be distinct. 


\begin{tabular}{lll}
\hline Name & Notation & Example $\begin{array}{r}\psi=[\neg P(x, y, z) \vee \neg Q(y, A) \\
\text { except }\{x=A\},\{x=C, y=D\}]\end{array}$ \\
\hline main clause & $\mathcal{M}(\psi)$ & $\neg P(x, y, z) \vee \neg Q(y, A)$ \\
main part & {$[\mathcal{M}(\psi)]$} & {$[\neg P(x, y, z) \vee \neg Q(y, A)]$} \\
variables & $\mathcal{V}(\psi)$ & $\{x, y, z\}$ \\
exceptions & $\Sigma(\psi)$ & $\{\{x=A\},\{x=C, y=D\}\}$ \\
exception clauses & $\mathcal{E}_{1}(\psi)$ & $\neg P(A, y, z) \vee \neg Q(y, A)$ \\
& $\mathcal{E}_{2}(\psi)$ & $\neg P(C, D, z) \vee \neg Q(D, A)$ \\
exception forms & $\mathcal{E}(\psi)$ & $\{[\neg P(A, y, z) \vee \neg Q(y, A)],[\neg P(C, D, z) \vee \neg Q(D, A)]\}$ \\
\hline
\end{tabular}

Figure 1: Summary of $\psi$-form notation.

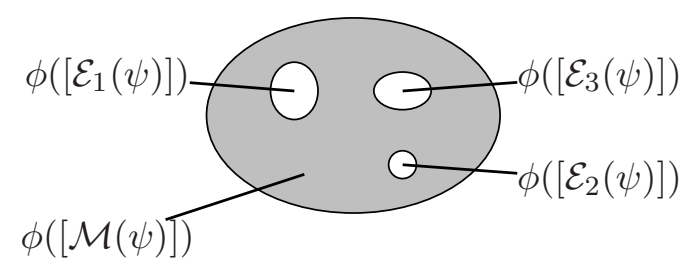

Figure 2: The set of clauses defined by a $\psi \cdot \phi(\psi)$ is depicted as the gray area and consists of all clauses of the main part $[\mathcal{M}(\psi)]$ that are not exceptions, i.e. are not in any of $\left[\mathcal{E}_{1}(\psi)\right], \ldots,\left[\mathcal{E}_{3}(\psi)\right]$. The main part $[\mathcal{M}(\psi)]$ contains the superset of all clauses of $\psi$.

2.2. $\psi$-forms as Sets. A $\psi$-form is a representation of a possibly infinite set of ground clauses. Here and throughout the paper, clauses that consist of the same set of literals are considered equal. The logical equivalence of such clauses allows us to disregard the order of their literals.

We define the set of ground clauses represented by a $\psi$-form $\psi$, called a $\psi$-set and denoted $\phi(\psi)$, as follows:

(1) When $\psi$ is simple, the set defined by $\psi$ consists of all ground instantiations of the main clause

$$
\phi(\psi)=\{\mathcal{M}(\psi) \sigma \mid \mathcal{M}(\psi) \sigma \text { is ground }\}
$$

This definition implies $\phi([c])=\{c\}$, when $c$ is a ground clause.

(2) When $\psi$ is not simple, the set defined by $\psi$ consists of all ground instantiations of the main clause minus the set of all ground instantiations of exception clauses.

$$
\phi(\psi)=\phi([\mathcal{M}(\psi)])-\phi\left(\left[\mathcal{E}_{1}(\psi)\right]\right)-\ldots-\phi\left(\left[\mathcal{E}_{n}(\psi)\right]\right),
$$

where $n=\|\Sigma(\psi)\|$. Any clause in the set $\phi\left(\left[\mathcal{E}_{1}(\psi)\right]\right) \cup \ldots \cup \phi\left(\left[\mathcal{E}_{n}(\psi)\right]\right)$ is called an exception of $\psi$.

Figure 2 illustrates the definition of a $\psi$-set.

To combine and compare $\psi$-sets represented by different $\psi$-forms, we introduce set operations and define the resulting $\psi$-set as follows. 
(1) For a set of $\psi$-forms $\left\{\psi_{1}, \ldots, \psi_{k}\right\}$ their $\psi$-set is the union of the $\psi$-sets of its elements.

$$
\phi\left(\left\{\psi_{1}, \ldots, \psi_{k}\right\}\right)=\cup_{i=1}^{k} \phi\left(\psi_{i}\right),
$$

(2) An expression $\square_{1} * \square_{2}$, where $\square_{1}$ and $\square_{2}$ denote either a single $\psi$-form or a set of $\psi$ forms, and $*$ denotes any of the set operations $\cap, \cup,-, \triangleright$ or $\underline{e}$ (last two operations are defined in the next section) represents a set of ground clauses obtained by applying the ${ }^{*}$ operation to the corresponding $\psi$-sets.

$$
\phi\left(\square_{1} * \square_{2}\right)=\phi\left(\square_{1}\right) * \phi\left(\square_{2}\right) .
$$

(3) Let $A$ and $B$ be $\psi$-forms or $\psi$-form expressions. We write $A=B$ and call $A$ and $B$ equivalent if and only if $\phi(A)=\phi(B)$, in other words, the sets of ground clauses represented by each $\psi$-form or expression are the same.

For example, the statement $\psi_{1}=\psi_{2} \cap \psi_{3}$ represents the equivalence of two $\psi$-sets: $\phi\left(\psi_{1}\right)$ and $\phi\left(\psi_{2} \cap \psi_{3}\right)$. The latter, in turn, according to definition (2.5) denotes the intersection $\phi\left(\psi_{2}\right) \cap \phi\left(\psi_{3}\right)$

2.2.1. $\psi$-set Membership. We say that a ground clause $c$ is in $\square$, written $c \in \square$ instead of $c \in \phi(\square)$.

Thus, according to (2.3), given a $\psi$-form $\psi$, a ground clause $c$ is in $\psi$ if and only if it can be obtained by instantiating the main clause, $\mathcal{M}(\psi)$, with some ground substitution $\sigma$, and cannot be obtained by instantiating any of $\psi$ 's exception clauses $\mathcal{E}_{1}(\psi), \ldots, \mathcal{E}_{n}(\psi)$.

$$
c \in \psi \text { iff } \exists \sigma . c=\mathcal{M}(\psi) \sigma \text { and } \forall \theta, i .1 \leq i \leq n \Longrightarrow c \neq \mathcal{E}_{i}(\psi) \theta
$$

We also define membership of a clause in a set of $\psi$-forms $\Psi$ in the obvious way:

$$
c \in \Psi \text { iff } \exists \psi \in \Psi . c \in \psi
$$

Deciding the membership of a ground clause in a simple $\psi$-form amounts to finding a substitution $\sigma$ that matches the literals in the main clause of the $\psi$-form with the literals of the clause. In a $\psi$-form with exceptions, after establishing membership in the main part, $[\mathcal{M}(\psi)]$, it is necessary to verify non-membership in the $\psi$-form's exception forms, all of which in turn are simple $\psi$-forms.

We define an operation set-match that computes such matching substitutions used to generate a clause from the main clause of a $\psi$-form. Given two clauses $a$ and $b$, where variables in $a$ and $b$ are distinct and denoted $V_{a}$ and $V_{b}$ respectively, we say that $a$ setmatches with $b$ if and only if there exists a substitution $\sigma$ on variables in $V_{a}$ such that $a \sigma=b$. The set of all most general such matching $\sigma$ 's is denoted $M G U_{\equiv}\left(a, b, V_{a}\right) .^{3}$

Example 2.1 below demonstrates that there can be more than one way a $\psi$-form clause can set-match with a ground clause.

Example 2.1. Let $\psi=[\neg P(x) \vee \neg P(y)$ except $\{\{x=A\}\}]$ and let $c=\neg P(A) \vee \neg P(B)$. There are two substitutions $\sigma_{1}=\{x=A, y=B\}$ and $\sigma_{2}=\{x=B, y=A\}$ such that $\mathcal{M}(\psi) \sigma_{1}=\mathcal{M}(\psi) \sigma_{2}=c$. However, $c$ can also be generated by instantiating the exception clause $\mathcal{E}_{1}(\psi)=\neg P(A) \vee \neg P(y)$ with substitution $\{y=B\}$, therefore $c \notin \psi$.

\footnotetext{
${ }^{3}$ Two substitutions $\sigma_{1}$ and $\sigma_{2}$ are equal if and only if the two can be made identical by consistently renaming variables in both. Thus $M G U_{\equiv}\left(a, b, V_{a}\right)$ does not include two equivalent substitutions.
} 
The possibility of multiple different instantiations producing the same ground clause complicates the reasoning with $\psi$-forms of this type, as even deciding membership of a ground clause in a $\psi$-form becomes somewhat more problematic compared to the case of $\psi$-forms for which each ground clause is generated with a unique substitution. Essentially, when each ground clause is obtained with a unique instantiation of the main form, checking $c \in \psi$ amounts to computing a set-match $M G U_{\equiv}(\mathcal{M}(\psi), c, \mathcal{V}(\psi))$ and, in case the setmatch results in a substitution $\sigma$, checking that $\sigma$ is not a superset of any substitution in $\psi$ 's exceptions $\Sigma(\psi)$. When, as in the Example 2.1. $M G U_{\equiv}(\mathcal{M}(\psi), c, \mathcal{V}(\psi))$ consists of more than a single substitution, we must consider all such substitutions in relation to the exceptions. To avoid the increase in the complexity of reasoning we introduce a notion of a fixed length $\psi$-form.

A $\psi$-form is called fixed length if and only if no two literals of the main clause unify when the variables in both literals are renamed to be distinct. Thus, $[\neg P(x, A) \vee \neg P(B, x)]$ is not a fixed length $\psi$-form, because $\neg P\left(x_{1}, A\right)$ and $\neg P\left(B, x_{2}\right)$ are unifiable. On the other hand, $[\neg P(x, A) \vee \neg P(x, B)]$ and $[\neg P(x, A) \vee \neg Q(y)]$ are examples of fixed length $\psi$-forms.

Observation 2.2. When $\psi$ is a fixed length $\psi$-form, there is a unique $\sigma$ for each clause $c \in \psi$ such that $\mathcal{M}(\psi) \sigma=c$.

Proof. The proof is by contradiction. Assuming there is a ground clause that is generated by more than one substitution on $\mathcal{M}(\psi)$, it is possible to construct a unifier for two literals of the main clause.

Other important properties of fixed length $\psi$-forms ensuring reduced complexity of reasoning are discussed in the end of Section 3.6.

Thus everywhere except for general $\psi$-form entailment theorems in Section 3.3 we limit our attention to fixed length $\psi$-forms.

A $\psi$-form is called well-formed if and only if it has no redundant exception forms, i.e. there is no subsumption between any two exception clauses. Any $\psi$-form can be reduced to a well-formed equivalent; henceforth, we only consider well-formed $\psi$-forms. The reduction procedure is simple and consists of examining pairs of different substitutions $\sigma_{i}, \sigma_{j}$ of $\Sigma(\psi)$. If $\sigma_{i} \subseteq \sigma_{j}$, then (and only then) $\left[\mathcal{E}_{j}(\psi)\right] \subseteq\left[\mathcal{E}_{i}(\psi)\right]$, and so we remove $\sigma_{j}$ from $\Sigma(\psi)$ and vice versa. In the worst case we will need to examine $E(E-1) / 2$ pairs.

2.3. $\psi$-form Logic. An interpretation or world is a triple (D, M, A), where D is a domain, $\mathrm{M}$ is a mapping between the constants of the language and the domain objects, and $A$ is a truth assignment on all ground atoms of the language. We limit worlds to those with infinite domains. We further assume each constant denotes a distinct domain object. A model of a proposition is a world that assigns true to that formula.

When $s$ is a proposition or a set of propositions and $w$ is a world, we write $w(s)$ if and only if $w(s)$ is true in $w$. For a set of propositions to be true in $w$, each element must be true in $w$. We write $I(s)$ to denote the set of all models of $s$, i.e. $I(s)=\{w \mid w(s)\}$.

We use the standard rules regarding the interpretation of atoms, negation and logical connectives. A $\psi$-form or a set of $\psi$-forms, denoted below by $\square$, is interpreted as (a possibly infinite) conjunction of all ground clauses it represents, and therefore

$$
I(\square)=\bigcap_{c \in \phi(\square)} I(c) .
$$


Now that we have defined an interpretation for $\psi$-forms we can define entailment in a logical language containing $\psi$-forms in the usual way. A formula $a$ entails a formula $b$, denoted $a \models b$, if and only if every model of $a$ is also a model of $b$, i.e. $I(a) \subseteq I(b)$.

We first examine the entailment between two ground clauses of negated literals. We write $c_{1} \subseteq c_{2}$ when a set of literals of the clause $c_{1}$ is a subset of the set of literals of the clause $c_{2}$. It is easy to see, that given two non-empty grownd clauses of negated literals $c_{1}$ and $c_{2}, c_{1} \models c_{2}$ if and only if $c_{1} \subseteq c_{2}$. Further, observe, that when $\square_{1}$ and $\square_{2}$ are two $\psi$-forms or sets of $\psi$-forms $\square_{1}=\square_{2}$ if and only if $\square_{1} \models \square_{2}$ and $\square_{2} \models \square_{1}$.

Note as well, that $\psi=\left[Q(\vec{x})\right.$ except $\left.\left\{\sigma_{1}, \ldots, \sigma_{n}\right\}\right]$ can be equivalently written as a first order formula that universally quantifies the variables of $\psi$ :

$$
\forall \vec{x} . Q(\vec{x}) \vee c_{1} \vee \ldots \vee c_{n},
$$

where each $c_{i}$ is an equality constraint obtained from $\sigma_{i}$, for instance if $\sigma_{i}=\{x=A, y=B\}$ then $c_{i}=(x=A \wedge y=B)$. We therefore call non-singleton $\psi$-forms quantified.

\section{Calculus of $\psi$-FORMS}

In this section we present the calculus of $\psi$-forms. We first demonstrate how subset, intersection and set-difference between $\psi$-forms are computed in simple cases. These operations lay the foundation for algorithms that compute entailment, as well as the computation of image and e-difference operations, which we define here. These operations are essential parts of reasoning and planning with $\psi$-forms. For example, they are used in PSIPOP ([5]) and PSIGraph ([12]) planners to determine if an effect of an action can bring about or undo a goal. E-difference is also used in the PSIPLAN's state update computation (4.6), presented later in this paper. Sound and complete methods of computing entailment, image and e-difference of fixed length $\psi$-forms are presented in the form of theorems that are easily convertible to algorithms. We summarize the complexity of computing entailment, image and e-difference between $\psi$-forms in PSIPLAN.

3.1. Operations $\subseteq, \cap$ and -. The operations subset, intersection and set-difference between $\psi$-forms are defined in the obvious way: for any ground clause $c$

$$
c \in \square_{1} * \square_{2} \text { if and only if } c \in \phi\left(\square_{1}\right) * \phi\left(\square_{2}\right)
$$

where $\square$ represents either a single $\psi$-form or a set of $\psi$-forms, and $*$ represents any of the operations $\subseteq, \cap$ or - . Calculation of $\subseteq, \cap$ and - is straightforward for simple fixed length $\psi$-forms.

$\left[\mathcal{M}\left(\psi_{1}\right)\right] \subseteq\left[\mathcal{M}\left(\psi_{2}\right)\right]$ if and only if all of $\left[\mathcal{M}\left(\psi_{1}\right)\right]$ 's clauses are also clauses of $\left[\mathcal{M}\left(\psi_{2}\right)\right]$. This requires that the main clause $\mathcal{M}\left(\psi_{2}\right)$ set-matches onto $\mathcal{M}\left(\psi_{1}\right)$ with some substitution $\sigma$. When this is true, for every ground substitution $\sigma_{1}$ on the variables of $\psi_{1}$, there is a ground substitution $\sigma_{2}=\sigma \sigma_{1}$ on $\mathcal{M}\left(\psi_{2}\right)$ such that $\mathcal{M}\left(\psi_{1}\right) \sigma_{1}=\mathcal{M}\left(\psi_{2}\right) \sigma_{2}$. Thus, for any $\psi_{1}$ and $\psi_{2}$, checking whether or not $\left[\mathcal{M}\left(\psi_{1}\right)\right] \subseteq\left[\mathcal{M}\left(\psi_{2}\right)\right]$ amounts to computing $M G U_{\equiv}\left(\mathcal{M}\left(\psi_{2}\right), \mathcal{M}\left(\psi_{1}\right), \mathcal{V}\left(\psi_{2}\right)\right)$.

$\left[\mathcal{M}\left(\psi_{1}\right)\right] \cap\left[\mathcal{M}\left(\psi_{2}\right)\right]$ is defined by all ground substitutions $\sigma_{g}$ such that $\mathcal{M}\left(\psi_{1}\right) \sigma_{g}=$ $\mathcal{M}\left(\psi_{2}\right) \sigma_{g}$. The set of most general $\sigma$ 's for which $\mathcal{M}\left(\psi_{1}\right) \sigma=\mathcal{M}\left(\psi_{2}\right) \sigma$ defines a set of main clauses that generate the $\psi$-forms denoting the intersection $\left[\mathcal{M}\left(\psi_{1}\right)\right] \cap\left[\mathcal{M}\left(\psi_{2}\right)\right]$. 
$\operatorname{Trans}(\sigma, \psi)$

- Returns part of $\sigma$ that binds variables of $\psi$ in exception-conformant format

1. In $\sigma$, replace all groups of bindings of the form $v_{1}=v, \ldots, v_{n}=v$, where $n>1, v_{1}, \ldots v_{n} \in \mathcal{V}(\psi)$, and $v \notin \mathcal{V}(\psi)$ with a set of bindings: $v_{1}=v_{n}, \ldots, v_{n-1}=v_{n}$.

2. Further, remove from $\sigma$ all bindings involving variables that are not in $\mathcal{V}(\psi)$.

3. Return $\sigma$

Figure 3: Procedure $\operatorname{Trans}(\sigma, \psi)$ transforms a substitution $\sigma$ into format suitable for the exceptions of $\psi$.

We say clause $a$ set-unifies with clause $b$ if and only if there exists a substitution $\sigma$ such that $a \sigma=b \sigma$, denoting the set of all most general such $\sigma$ 's by $M G U_{\equiv}(a, b)$. Thus, $\left[\mathcal{M}\left(\psi_{1}\right)\right] \cap\left[\mathcal{M}\left(\psi_{2}\right)\right]=\left\{\left[\mathcal{M}\left(\psi_{1}\right) \sigma\right] \mid \sigma \in M G U_{\equiv}\left(\mathcal{M}\left(\psi_{1}\right), \mathcal{M}\left(\psi_{2}\right)\right)\right\}$.

The intersection of the main parts of two $\psi$-forms $\psi_{1}$ and $\psi_{2}$ consists of the clauses denoted equivalently by each of the two sets of $\psi$-forms

$$
\begin{aligned}
& \left\{\left[\mathcal{M}\left(\psi_{1}\right) \sigma\right] \mid \sigma \in M G U_{\equiv}\left(\mathcal{M}\left(\psi_{1}\right), \mathcal{M}\left(\psi_{2}\right)\right)\right\}, \text { and } \\
& \left\{\left[\mathcal{M}\left(\psi_{2}\right) \sigma\right] \mid \sigma \in M G U_{\equiv}\left(\mathcal{M}\left(\psi_{1}\right), \mathcal{M}\left(\psi_{2}\right)\right)\right\} .
\end{aligned}
$$

For example, when $\psi_{1}=[\neg P(x, A)]$ and $\psi_{2}=[\neg P(B, y)], \quad M G U_{\equiv}\left(\mathcal{M}\left(\psi_{1}\right), \mathcal{M}\left(\psi_{2}\right)\right)=$ $\{\{x=B, y=A\}\}$ and $\psi_{1} \cap \psi_{2}$ equals $\{\neg P(B, A)\}$.

When $\psi_{1}$ is simple and is a subset of $\left[\mathcal{M}\left(\psi_{2}\right)\right]$ and both $\psi$-forms are fixed length, subtracting $\psi_{1}$ from $\psi_{2}$ is a matter of adding a substitution $\sigma=M G U_{\equiv}\left(\mathcal{M}\left(\psi_{2}\right), \mathcal{M}\left(\psi_{1}\right), \mathcal{V}\left(\psi_{2}\right)\right)$, which generates $\psi_{1}$ from $\mathcal{M}\left(\psi_{2}\right)$, to $\Sigma\left(\psi_{2}\right)$. Indeed, $\left[\mathcal{M}\left(\psi_{2}\right) \sigma\right]$ equals $\psi_{1}$, thus, by adding $\sigma$ to $\Sigma\left(\psi_{2}\right)$ we are subtracting from $\psi_{2}$ the clauses of $\psi_{1}$. As a matching substitution, $\sigma$ may contain bindings on variables of $\psi_{2}$ either to variables of $\psi_{1}$ or to constants. Procedure Trans $\left(\sigma, \psi_{2}\right)$, presented in Figure 3, generates an equivalent substitution, which uses only variables from $\mathcal{V}\left(\psi_{2}\right)$, as only those variables can appear in the set of $\psi_{2}$ 's exceptions.

Figure 4 contains examples of $\psi$-form calculations described to this point.

When $\psi_{1}$ is simple, and both $\psi_{1}$ and $\psi_{2}$ are fixed length, but $\psi_{1}$ is not necessarily a subset of $\left[\mathcal{M}\left(\psi_{2}\right)\right]$, the computation of $\psi_{2}-\psi_{1}$ is reduced to the previous case by observing that $\psi_{2}-\psi_{1}=\psi_{2}-\left(\left[\mathcal{M}\left(\psi_{2}\right)\right] \cap\left[\mathcal{M}\left(\psi_{1}\right)\right]\right)$, since $\left[\mathcal{M}\left(\psi_{2}\right)\right] \cap\left[\mathcal{M}\left(\psi_{1}\right)\right]$ is a subset of $\left[\mathcal{M}\left(\psi_{2}\right)\right]$. When $\left[\mathcal{M}\left(\psi_{1}\right)\right] \cap\left[\mathcal{M}\left(\psi_{2}\right)\right]$ is empty, then $\psi_{2}-\psi_{1}$ is just $\psi_{2}$.

Operations of $\cap,-$ and $\subseteq$ for two simple fixed length $\psi$-forms and membership of a clause in any $\psi$-form each take constant bounded time when the number of clauses, variables and exceptions in a $\psi$-form are constant bounded.

3.2. E-Difference and Image. To capture the relations between parts of $\psi$-forms necessary to formulate the $\psi$-form entailment theorem, we introduce two new operations: the image, denoted $\psi_{1} \triangleright \psi_{2}$, is the subset of $\psi_{2}$ that is entailed by $\psi_{1}$, and the e-difference, denoted $\psi_{2} \underline{e} \psi_{1}$, is the subset of $\psi_{2}$ that is not entailed by $\psi_{1}$. Thus, $\left(\psi_{2} \underline{e} \psi_{1}\right)$ and $\left(\psi_{1} \triangleright \psi_{2}\right)$ always partition $\psi_{2}$.

Formally, for any two sets of ground propositions $A$ and $B$, e-difference and image are defined respectively as follows.

$$
\begin{aligned}
& B \stackrel{e}{e}=\{b \mid b \in B \wedge A \not \models b\}, \\
& A \triangleright B=\{b \mid b \in B \wedge A \models b\} .
\end{aligned}
$$




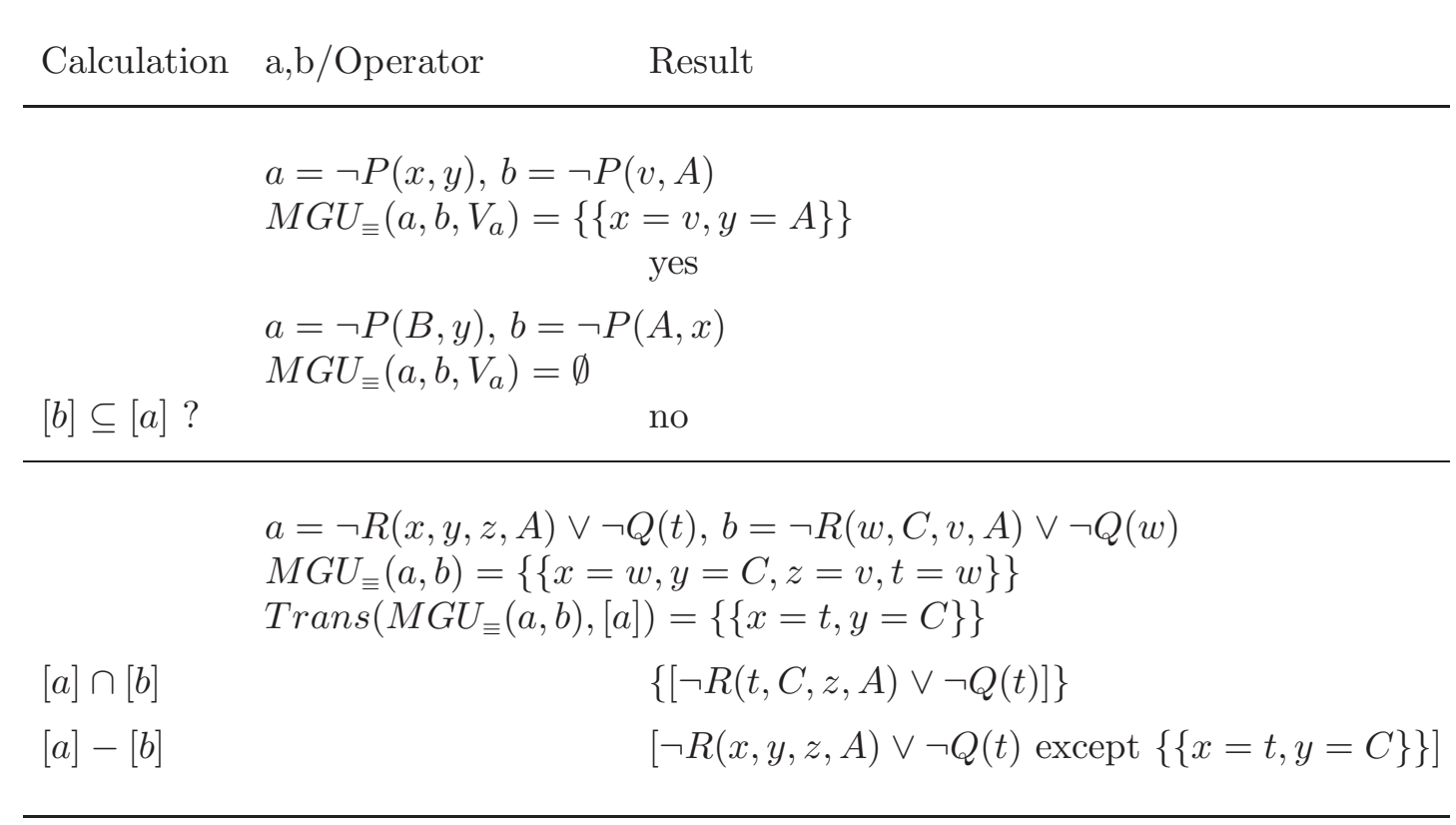

Figure 4: Examples of the calculus computations involving set-match (p. 8) and setunification (p. 11]) operators on simple $\psi$-forms [a] and [b].

Example 3.1. Let $A=\{p, \neg q\}$ and $B=\{p, r, \neg q \vee \neg v\}$. Then, $A \triangleright B=\{p, \neg q \vee \neg v\}$ and $B \underline{e} A=\{r\}$.

The following equivalences trivially follow from the definitions.

$$
\begin{aligned}
& B \stackrel{e}{-} A=B-(A \triangleright B) \\
& A \triangleright B=B-(B \stackrel{e}{e} A)
\end{aligned}
$$

As we show later in this section, the operations $\triangleright$ and $\underline{e}$ applied to fixed length $\psi$-forms produce sets of clauses that can always be represented by a finite set of fixed length $\psi$-forms.

3.3. Entailment. The next Theorem establishes the fact that a set of $\psi$-forms $\Psi$ entails another $\psi$-form $\psi$ if and only if for each clause of $\psi$ there exists a clause in $\Psi$ entailing it. The intuition behind this observation is: any two negated ground clauses $c_{1}$ and $c_{2}$ conjoined entail the same set of negated ground clauses as the union of clauses entailed by each of $c_{1}$ and $c_{2}$ separately.

Theorem 3.2. Given a set of $\psi$-forms $\Psi=\left\{\psi_{1}, \ldots, \psi_{n}\right\}$ and a $\psi$-form $\psi, \Psi \models \psi$ if and only if for every ground clause $c \in \psi$ there exists a ground clause $c^{\prime} \in \Psi$ such that $c^{\prime} \models c$.

Proof. $(\Rightarrow)$ A proof by contradiction is straightforward and thus omitted.

$(\Leftarrow)$ Trivially follows from the definition of entailment. 


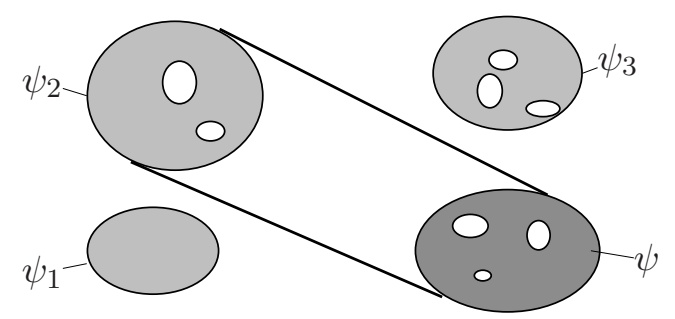

Figure 5: For a set of $\psi$-forms $\left\{\psi_{1}, \psi_{2}, \psi_{3}\right\}$ to entail another $\psi$-form $\psi$ there must be a $\psi$-form in the set ( $\psi_{2}$ in the figure) whose main part entails the main part of $\psi$.

The property critical for the efficiency of $\psi$-form reasoning is formulated in Theorem 3.4 and depicted in Figure [5 given a set of $\psi$-forms $\Psi=\left\{\psi_{1}, \ldots, \psi_{n}\right\} \Psi \models \psi$ only if there is a $\psi$-form $\psi_{i} \in \Psi$ that nearly entails $\psi$, i.e. $\left[\mathcal{M}\left(\psi_{i}\right)\right] \models[\mathcal{M}(\psi)]$.

Nearly entailment is a necessary condition for $\psi$-form entailment. As follows from Theorem 3.3 entailment between two simple $\psi$-forms is a matter of finding what we call a subset-match, or subsumption of their main clauses.

Given two clauses $a$ and $b$, where variables in $a$ and $b$ are distinct and denoted $V_{a}$ and $V_{b}$ respectively, we say that $a$ subsumes $b$ (or $a$ subset-matches $b$ ) if and only if there exists a substitution $\sigma$ on variables in $V_{a}$ such that $a \sigma \subseteq b$. We denote by $M G U_{\subseteq}\left(a, b, V_{a}\right)$ the set of all such $\sigma$ 's.

Note that there can be more than one way a clause can subsume another clause. For example, matching $a=P(x, y)$ onto $b=P(z, D) \vee Q(D, E) \vee P(A, B)$, produces two different substitutions: $\{x=z, y=D\}$ and $\{x=A, y=B\}$, and hence $M G U_{\subseteq}(a, b,\{x, y\})=$ $\{\{x=z, y=D\},\{x=A, y=B\}\}$.

Theorem 3.3. Given two simple $\psi$-forms, $\psi_{1}$ and $\psi_{2}, \psi_{1} \models \psi_{2}$ if and only if the main clause of $\psi_{1}$ subsumes the main clause of $\psi_{2}$, i.e. there exists a substitution $\sigma$ such that $\mathcal{M}\left(\psi_{1}\right) \sigma \subseteq \mathcal{M}\left(\psi_{2}\right)$ and consequently $\operatorname{MGU}_{\subseteq}\left(\mathcal{M}\left(\psi_{1}\right), \mathcal{M}\left(\psi_{2}\right), \mathcal{V}\left(\psi_{1}\right)\right) \neq \emptyset$.

Proof. $(\Rightarrow)$ Given $\psi_{1} \models \psi_{2}$, suppose that

$$
\mathcal{M}\left(\psi_{1}\right)=\left\{\neg Q_{1}\left(\vec{x}_{1}\right), \ldots, \neg Q_{n}\left(\vec{x}_{n}\right)\right\} \quad \text { and } \quad \mathcal{M}\left(\psi_{2}\right)=\left\{\neg P_{1}\left(\vec{y}_{1}\right), \ldots, \neg P_{k}\left(\vec{y}_{k}\right)\right\} .
$$

Assume $M G U_{\subseteq}\left(\mathcal{M}\left(\psi_{1}\right), \mathcal{M}\left(\psi_{2}\right), \mathcal{V}\left(\psi_{1}\right)\right)=\emptyset$.

This means that for any substitution $\sigma$ on variables of $\psi_{1}$, there exists a literal in $\mathcal{M}\left(\psi_{1}\right) \sigma$, which does not match any of $\left\{\neg P_{1}\left(\vec{y}_{1}\right), \ldots, \neg P_{k}\left(\vec{y}_{k}\right)\right\}$. We can assume without any loss of generality that the mismatched literal is $\neg Q_{1}\left(\vec{x}_{1}\right)$. Note that the mismatch can occur due to one of the following reasons (we also call them mismatch types):

(1) the predicate denoted by $Q_{1}$ is not the same as denoted by $P_{i}$,

(2) the argument list of $Q_{1}\left(\vec{x}_{1}\right)$ has a constant, call it a matching value, at the position where $P_{i}\left(\vec{y}_{i}\right)$ 's argument list has a variable, call it, a mismatched variable.

(3) the argument list of $Q_{1}\left(\vec{x}_{1}\right)$ has a constant, call it $B_{i}$, at the position where $P_{i}\left(\vec{y}_{i}\right)$ has a different constant, $C_{i}$.

We now construct a clause from $\psi_{2}$ and show that it cannot contain as a subclause any clause of $\psi_{1}$. According to Theorem 3.2 we would then contradict the fact that $\psi_{1} \models \psi_{2}$.

Let $P^{\prime}$ be an instance of $\mathcal{M}\left(\psi_{2}\right)$, which is obtained by assigning to each variable in $\mathcal{V}\left(\psi_{2}\right)$ a constant value which does not occur anywhere in $\mathcal{M}\left(\psi_{1}\right)$. Since the number of constants in the language is infinite, this can always be done. Since $\mathcal{M}\left(\psi_{1}\right)$ does not subset 
match onto $\mathcal{M}\left(\psi_{2}\right)$, it surely does not subset match onto $P^{\prime}$, because for any substitution $\sigma$ on $\mathcal{V}\left(\psi_{1}\right)$ the number of mismatches between any $Q$ and any literal of $P^{\prime}$ is at least the same as the number of mismatches between a $Q$ and a $P$ in $\mathcal{M}\left(\psi_{2}\right)$, or higher, because of the new mismatches of type 3. Therefore, there is no instance of $\mathcal{M}\left(\psi_{1}\right)$ that is a subclause of $P^{\prime}$.

$(\Leftarrow)$ The existence of $\sigma$ such that $\mathcal{M}\left(\psi_{1}\right) \sigma \subseteq \mathcal{M}\left(\psi_{2}\right)$ means that for every ground clause $c_{2}$ of $\psi_{2}$, assuming $c_{2}=\mathcal{M}\left(\psi_{2}\right) \sigma^{\prime}$, there is a clause $c_{1}$ in $\psi_{1}, c_{1}=\mathcal{M}\left(\psi_{1}\right) \sigma \sigma^{\prime}$, which is a subset of $c_{2}$, and therefore $\psi_{1} \models \psi_{2}$.

We proceed to the necessary condition for $\psi$-form entailment. Theorem 3.4 states that in order for a set of $\psi$-forms $\Psi$ to entail another $\psi$-form $\psi$, there must exist a $\psi$-form in $\Psi$ that nearly entails $\psi$, i.e. whose main part entails the main part of $\psi$.

Theorem 3.4. Given a set of $\psi$-forms $\Psi=\left\{\psi_{1}, \ldots, \psi_{n}\right\}$ and a $\psi$-form $\psi, \Psi \models \psi$ only if there is a $\psi$-form $\psi_{i}$ in $\Psi$ such that $\left(\left[\mathcal{M}\left(\psi_{i}\right)\right] \models[\mathcal{M}(\psi)]\right)$.

Proof. We construct a clause of $[\mathcal{M}(\psi)]$ and show that if none of $\left[\mathcal{M}\left(\psi_{1}\right)\right], \ldots,\left[\mathcal{M}\left(\psi_{n}\right)\right]$ entail it, then $\left\{\psi_{1}, \ldots, \psi_{n}\right\}$ does not entail $\psi$.

Suppose none of $\left[\mathcal{M}\left(\psi_{1}\right)\right], \ldots,\left[\mathcal{M}\left(\psi_{n}\right)\right]$ entail $[\mathcal{M}(\psi)]$. Therefore according to Theorem 3.3 none of the main parts of these $\psi$-forms subset match onto $\mathcal{M}(\psi)$. Let $\sigma$ be a substitution on $\mathcal{V}(\psi)$ that assigns to each variable a constant value that does not occur in any of $\psi_{1}, \ldots, \psi_{n}$, nor in the exceptions of $\psi$. This is always possible due to infinite number of constants in the language. None of the clauses in $\mathcal{M}\left(\psi_{1}\right), \ldots, \mathcal{M}\left(\psi_{n}\right)$ subset match onto $c=\mathcal{M}(\psi) \sigma$, because none of the main clauses of these $\psi$-forms subsume $\mathcal{M}(\psi)$ and the constants of $\sigma$ do not appear in any of $\left[\mathcal{M}\left(\psi_{1}\right)\right], \ldots,\left[\mathcal{M}\left(\psi_{n}\right)\right]$. Thus, the clause $c=\mathcal{M}(\psi) \sigma$ of $\psi$ is not entailed by any clause in $\left\{\left[\mathcal{M}\left(\psi_{1}\right)\right], \ldots,\left[\mathcal{M}\left(\psi_{n}\right)\right]\right\}$. Since $\Phi \subseteq\left\{\left[\mathcal{M}\left(\psi_{1}\right)\right], \ldots,\left[\mathcal{M}\left(\psi_{n}\right)\right]\right\}$, according to Theorem 3.2 we conclude that $\Psi \not \forall \psi$. We arrive at a contradiction.

The next example demonstrates that Theorem 3.4 contains a necessary but not sufficient condition for the $\psi$-form entailment, and further motivates the operations of image and edifference.

Example 3.5. Consider two $\psi$-forms $\psi_{1}$ and $\psi_{2}$ below.

$$
\begin{aligned}
& \psi_{1}=[\neg \operatorname{In}(x, \text { Box } 1) \vee \neg \text { Fragile }(x) \text { except }\{\{x=\text { Wine }\}\}] \\
& \psi_{2}=[\neg \operatorname{In}(y, \text { Box } 1) \vee \neg \text { Fragile }(y) \vee \neg \text { Owner }(y, \text { Joe })]
\end{aligned}
$$

Here, $\operatorname{In}(x, y)$ states that $x$ is in $y, \operatorname{Fragile}(x)$ denotes that $x$ is a fragile item, and Owner $(x, y)$ denotes that $x$ 's owner is $y$. Thus, $\psi_{1}$ states that there are no fragile items in Box1 except for possibly a bottle of wine. $\psi_{2}$ states that there are no fragile items in Box1 that are owned by Joe. Notice that $\psi_{2}$ is simple and thus $\psi_{2}=\left[\mathcal{M}\left(\psi_{2}\right)\right]$.

The main clause of $\psi_{1}$ subsumes the main clause of $\psi_{2}$, so $\psi_{1}$ nearly entails $\psi_{2}$. Therefore, the main part of $\psi_{1},\left[\mathcal{M}\left(\psi_{1}\right)\right]$ entails $\psi_{2}$, but because the exception of $\psi_{1}$ weakens it, $\psi_{1}$ does not entail $\psi_{2}$. In fact, $\psi_{1}$ entails all clauses of $\psi_{2}$ except for the clause $\neg \operatorname{In}($ Wine, Box 1$) \vee \neg$ Fragile (Wine) $\vee \neg$ Owner $($ Wine, Joe).

The only clause of $\psi_{2}$ that is not entailed by $\psi_{1}$ is $\neg \operatorname{In}($ Wine, Box 1$) \vee \neg$ Fragile $($ Wine $) \vee$ $\neg$ Owner (Wine, Joe), which is exactly the clause entailed by $\psi_{1}$ 's single exception, i.e. $\psi_{2} \stackrel{e}{{ }^{\prime}} \psi_{1}=\left[\mathcal{E}_{1}\left(\psi_{1}\right)\right] \triangleright \psi_{2}=[\neg \operatorname{In}($ Wine, Box 1$) \vee \neg$ Fragile $($ Wine $) \vee \neg$ Owner $($ Wine, Joe $)]$. 


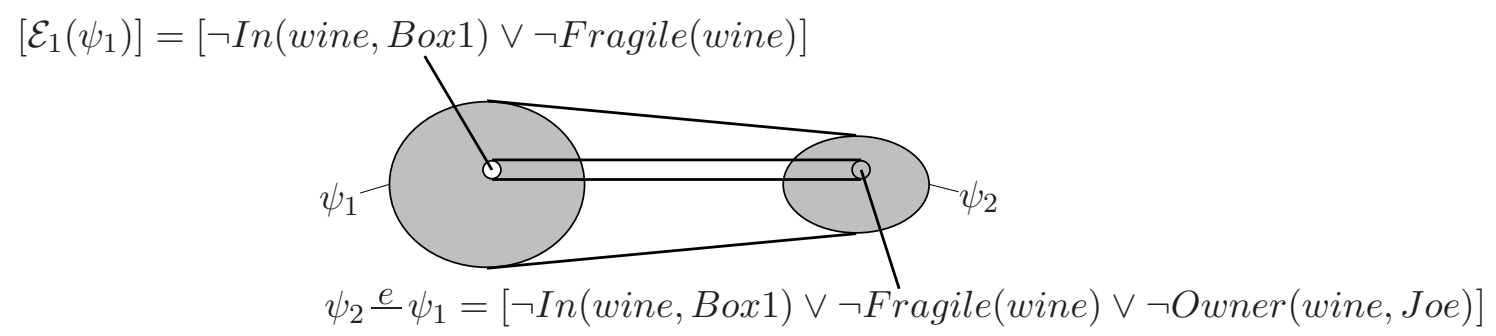

Figure 6: Illustrates Example 3.5. The small ellipse inside $\psi_{2}$ represents the only clause of $\psi_{2}$ not entailed by $\psi_{1}$, i.e. $\psi_{2} \frac{e}{-} \psi_{1}$. The area between the outer and the inner ellipsis is the image $\psi_{1} \triangleright \psi_{2}$.

The image $\psi_{1} \triangleright \psi_{2}$ is simply $\psi_{2}$ with a single exception added:

$\psi_{1} \triangleright \psi_{2}=[\neg \operatorname{In}(y$, Box 1$) \vee \neg$ Fragile $(y) \vee \neg$ Owner $(y$, Joe $)$ except $\{\{y=$ Wine $\}\}]$.

So, while $\psi_{1}$ nearly entails $\psi_{2}$, the e-difference $\psi_{2} \underline{e} \psi_{1}$ is not empty, i.e. $\psi_{1}$ does not entail $\psi_{2}$. This is illustrated in Figure 6.

Theorem 3.6. $\psi$-form Entailment. Let $\psi_{1}, \ldots, \psi_{n}$ and $\psi$ be arbitrary $\psi$-forms. $\left\{\psi_{1}, \ldots, \psi_{n}\right\} \models \psi$ if and only if there exists a $k, 1 \leq k \leq n$, such that:

- $\left[\mathcal{M}\left(\psi_{k}\right)\right] \models[\mathcal{M}(\psi)]$ (i.e., the main part of $\psi_{k}$ entails the main part of $\psi$ ), and

- $\left\{\psi_{1}, \ldots, \psi_{k-1}, \psi_{k+1}, \ldots, \psi_{n}\right\} \models \psi \underline{e} \psi_{k}$.

Proof. The first requirement of this Theorem follows from Theorem 3.4 While the main part of $\psi_{k}$ entails $\psi$, the exceptions of $\psi_{k}$ weaken $\psi_{k}$. Thus, each clause in $\psi \underline{e} \psi_{k}$ must be entailed by some other $\psi$-form in $\left\{\psi_{1}, \ldots, \psi_{k-1}, \psi_{k+1}, \ldots, \psi_{n}\right\}$.

Thus, in order for a set of $\psi$-forms $\Psi$ to entail another $\psi$-form $\psi$, there must exist a $\psi$-form $\psi_{k}$ in $\Psi$ that entails most of $\psi$, and the rest of $\psi$, i.e. $\psi \underline{e} \psi_{k}$ must be entailed by $\Psi$ without $\psi_{k}$.

We have formulated the necessary and sufficient conditions for $\psi$-form entailment using e-difference. We next present the methods of computing image and e-difference via simple operations of subset matching and unification, first for simple fixed length $\psi$-forms and then for fixed length $\psi$-forms with exceptions. Complexity bounds for the computation of entailment, image and e-difference appear in Section 3.6.

3.4. Simple Fixed Length $\psi$-forms. In this section, we present methods of computing the operations of $\psi$-form image and e-difference for two simple fixed length $\psi$-forms.

The image $\psi_{1} \triangleright \psi_{2}$ denotes a set of all clauses of $\psi_{2}$ that are entailed by $\psi_{1}$, i.e. all clauses of $\psi_{2}$ that have a subclause in $\psi_{1}$. Thus, when $\psi_{1}$ and $\psi_{2}$ are simple fixed length $\psi$ forms, computing $\psi_{1} \triangleright \psi_{2}$ reduces to instantiating $\mathcal{M}\left(\psi_{2}\right)$ with subset-unifying substitutions, i.e. substitutions $\sigma$ for which $\mathcal{M}\left(\psi_{1}\right) \sigma \subseteq \mathcal{M}\left(\psi_{2}\right) \sigma$. Formally, we say that $a$ subset-unifies with $b$ if and only if there exists a substitution $\sigma$ such that $a \sigma \subseteq b \sigma$, denoting the set of all most general such $\sigma$ 's by $M G U_{\subseteq}(a, b)$.

For example, consider $a=P(x, y), b=P(z, D) \vee Q(D, E) \vee P(A, B)$, and $c=$ $P(A, x)$. We have $M G U_{\subseteq}(a, b)=\{\{x=z, y=D\},\{x=A, y=B\}\}$ and $M G U_{\subseteq}(c, b)=$ $\{\{z=A, x=D\},\{x=B\}\}$. 
Calculation a,b/Operator Result

$\begin{aligned} & a=\neg P(x, A), b=\neg P(B, y) \vee \neg P(C, z) \vee \neg Q(y) \\ & M G U_{\subseteq}\left(a, b, V_{a}\right)=\emptyset \\ & \text { no } \\ & {[a] \models[b] ? \quad } \\ & M G U_{\subseteq}(a, b)=\{\{x=B, y=A\},\{x=C, z=A\}\} \\ &\{[\neg P(B, A) \vee \neg P(C, z) \vee \neg Q(A)], \\ & {[\neg P(B, y) \vee \neg P(C, A) \vee \neg Q(y)]\} } \\ & {[a] \triangleright[b] \quad } {[\neg P(B, y) \vee \neg P(C, z) \vee \neg Q(y) \operatorname{except~}\{\{y=A\},\{z=A\}\}] } \\ & {[b] \underline{e}[a] \quad }\end{aligned}$

Figure 7: Examples of the entailment, image and e-difference computations on simple $\psi$ formsThese computations utilize subset-match and subset-unification operators (defined on pages 13 and 15 respectively).

Theorem 3.7. Let $\psi_{1}$ and $\psi_{2}$ be simple fixed length $\psi$-forms.

$$
\psi_{1} \triangleright \psi_{2}=\left\{\left[\mathcal{M}\left(\psi_{2}\right) \sigma\right] \mid \sigma \in M G U_{\subseteq}\left(\mathcal{M}\left(\psi_{1}\right), \mathcal{M}\left(\psi_{2}\right)\right)\right\}
$$

Proof. It is easy to verify equality of the two sets by showing inclusion both ways.

Computing the e-difference, $\psi_{2} \frac{e}{-} \psi_{1}$, similar to the regular difference $\psi_{2}-\psi_{1}$ (page 111), amounts to adding exceptions to $\psi_{2}$. These exceptions represent the set of all clauses of $\left[\mathcal{M}\left(\psi_{2}\right)\right]$ entailed by $\left[\mathcal{M}\left(\psi_{1}\right)\right]$, i.e. the image $\left[\mathcal{M}\left(\psi_{1}\right)\right] \triangleright\left[\mathcal{M}\left(\psi_{2}\right)\right]$, and are obtained by computing the $M G U_{\subseteq}\left(\mathcal{M}\left(\psi_{1}\right), \mathcal{M}\left(\psi_{2}\right)\right)$.

Theorem 3.8. Let $\psi_{2}$ be an arbitrary fixed length $\psi$-form and $\psi_{1}$ be a simple fixed length $\psi$-form.

$$
\psi_{2} \stackrel{e}{e} \psi_{1}= \begin{cases}\emptyset, & \text { if } \psi_{1} \models \psi_{2}, \\ \left\{\left[\mathcal{M}\left(\psi_{2}\right) \text { except } \Sigma\left(\psi_{2}\right) \cup \Sigma^{\prime}\right]\right\}, & \text { otherwise, }\end{cases}
$$

where $\Sigma^{\prime}=\left\{\sigma^{\prime} \mid \sigma^{\prime}=\operatorname{Trans}\left(\sigma, \psi_{2}\right)\right.$, where $\left.\sigma \in M G U_{\subseteq}\left(\mathcal{M}\left(\psi_{1}\right), \mathcal{M}\left(\psi_{2}\right)\right)\right\}$. (Recall that Trans $\left(\sigma, \psi_{2}\right)$ defined in Figure 3 transforms substitution $\sigma$ to an equivalent one that conforms to the format of exceptions of $\psi_{2}$.)

Proof. To prove this theorem we use Theorem 3.7 and the equality $\psi_{2} \underline{e} \psi_{1}=\psi_{2}-\left(\psi_{1} \triangleright \psi_{2}\right)$. According to definition (2.3)

$$
\left[\mathcal{M}\left(\psi_{2}\right) \text { except } \Sigma\left(\psi_{2}\right) \cup \Sigma^{\prime}\right]=\left[\mathcal{M}\left(\psi_{2}\right)\right]-\mathcal{E}\left(\psi_{2}\right)-\left[\mathcal{M}\left(\psi_{2}\right) \sigma_{1}^{\prime}\right]-\ldots-\left[\mathcal{M}\left(\psi_{2}\right) \sigma_{n}^{\prime}\right],
$$

where $\Sigma^{\prime}=\left\{\sigma_{1}^{\prime}, \ldots, \sigma_{n}^{\prime}\right\}$. Note that $\left[\mathcal{M}\left(\psi_{2}\right)\right]-\mathcal{E}\left(\psi_{2}\right)=\psi_{2}$, and that $\left[\mathcal{M}\left(\psi_{2}\right) \sigma_{1}^{\prime}\right] \cup \ldots \cup$ $\left[\mathcal{M}\left(\psi_{2}\right) \sigma_{n}^{\prime}\right]=\psi_{1} \triangleright\left[\mathcal{M}\left(\psi_{2}\right)\right]$, and thus

$$
\left[\mathcal{M}\left(\psi_{2}\right) \text { except } \Sigma\left(\psi_{2}\right) \cup \Sigma^{\prime}\right]=\psi_{2}-\left(\psi_{1} \triangleright\left[\mathcal{M}\left(\psi_{2}\right)\right]\right) .
$$

It remains to show that

$$
\psi_{2}-\left(\psi_{1} \triangleright\left[\mathcal{M}\left(\psi_{2}\right)\right]\right)=\psi_{2}-\left(\psi_{1} \triangleright \psi_{2}\right) .
$$

Indeed $\left(\psi_{1} \triangleright \psi_{2}\right)=\left(\psi_{1} \triangleright\left[\mathcal{M}\left(\psi_{2}\right)\right]\right)-\left(\psi_{1} \triangleright \mathcal{E}\left(\psi_{2}\right)\right)$. Substituting the right hand side instead of $\left(\psi_{1} \triangleright \psi_{2}\right)$ in (3.3), we get

$$
\psi_{2}-\left(\psi_{1} \triangleright\left[\mathcal{M}\left(\psi_{2}\right)\right]\right)=\psi_{2}-\left[\left(\psi_{1} \triangleright\left[\mathcal{M}\left(\psi_{2}\right)\right]\right)-\left(\psi_{1} \triangleright \mathcal{E}\left(\psi_{2}\right)\right)\right]
$$


Since $\left(\psi_{1} \triangleright \mathcal{E}\left(\psi_{2}\right)\right)$ is in $\mathcal{E}\left(\psi_{2}\right)$ and therefore definitely not in $\psi_{2}$,

$$
\psi_{2}-\left(\psi_{1} \triangleright\left[\mathcal{M}\left(\psi_{2}\right)\right]\right)=\psi_{2}-\left[\left(\psi_{1} \triangleright\left[\mathcal{M}\left(\psi_{2}\right)\right]\right)-\left(\psi_{1} \triangleright \mathcal{E}\left(\psi_{2}\right)\right)\right]=\psi_{2}-\left(\psi_{1} \triangleright\left[\mathcal{M}\left(\psi_{2}\right)\right]\right)
$$

We have arrived at a tautology, which proves (3.3).

Note that the result of the e-difference may not be a well-formed $\psi$-form.

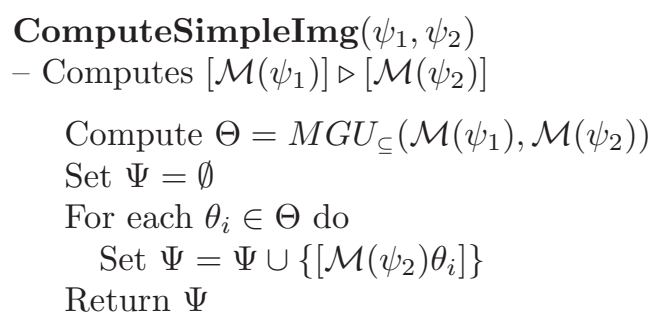

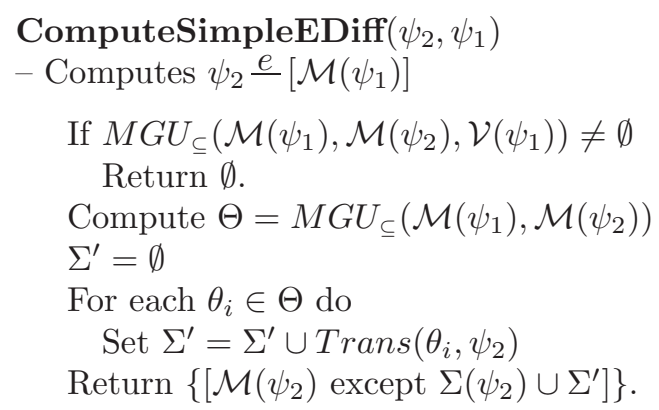

Figure 7 presents examples of computing image and e-difference between simple $\psi$ forms, and Figure 8 presents algorithms for these computations, based on Theorems 3.7 and 3.8

3.5. Arbitrary Fixed Length $\psi$-forms. In this section, we present methods of computing the operations of $\psi$-form image and e-difference for two arbitrary fixed length $\psi$-forms.

Let $\psi_{1}$ and $\psi_{2}$ be arbitrary fixed length $\psi$-forms. To find either the image or the edifference we first find the image of the main part of $\psi_{1}$ onto the main part of $\psi_{2}$. Since the exceptions of $\psi_{1}$ weaken it, we must then calculate the part of $\left[\mathcal{M}\left(\psi_{2}\right)\right]$ that is not entailed by $\psi_{1}$ due to the exceptions. We'll call this a set of "holes" ( denoted by $H\left(\psi_{1}, \psi_{2}\right)$ ).

Formally, we define set of holes $H\left(\psi_{1}, \psi_{2}\right)$ as follows

$$
H\left(\psi_{1}, \psi_{2}\right)=\left(\left[\mathcal{M}\left(\psi_{1}\right)\right] \triangleright\left[\mathcal{M}\left(\psi_{2}\right)\right]\right)-\left(\psi_{1} \triangleright\left[\mathcal{M}\left(\psi_{2}\right)\right]\right),
$$

i.e. holes are parts of $\left[\mathcal{M}\left(\psi_{2}\right)\right]$ that are entailed by $\left[\mathcal{M}\left(\psi_{1}\right)\right]$, but not by $\psi_{1}$.

Image and e-difference operations are easily formulated using $H\left(\psi_{1}, \psi_{2}\right)$. The image of $\psi_{1}$ onto $\psi_{2}$ consists of clauses of the main part of $\psi_{2}$ entailed by the main part of $\psi_{1}$, i.e. $\left[\mathcal{M}\left(\psi_{1}\right)\right] \triangleright\left[\mathcal{M}\left(\psi_{2}\right)\right]$, minus the set of holes $H\left(\psi_{1}, \psi_{2}\right)$ and minus $\psi_{2}$ 's own exceptions. Similarly, the e-difference $\psi_{2} \underline{e} \psi_{1}$ consists of the part of the main part of $\psi_{2}$, $\left[\mathcal{M}\left(\psi_{2}\right)\right]$, not entailed by $\left[\mathcal{M}\left(\psi_{1}\right)\right]$, i.e. $\left[\mathcal{M}\left(\psi_{2}\right)\right] \stackrel{e}{e}\left[\mathcal{M}\left(\psi_{1}\right)\right]$ plus the set of holes $H\left(\psi_{1}, \psi_{2}\right)$, minus the set of $\psi_{2}$ 's exceptions. These two facts are presented in the following two Lemmas.

Lemma 3.9. $\psi_{1} \triangleright \psi_{2}=\left(\left(\left[\mathcal{M}\left(\psi_{1}\right)\right] \triangleright\left[\mathcal{M}\left(\psi_{2}\right)\right]\right)-H\left(\psi_{1}, \psi_{2}\right)\right)-\mathcal{E}\left(\psi_{2}\right)$.

Proof. Let $A=\left[\mathcal{M}\left(\psi_{1}\right)\right] \triangleright\left[\mathcal{M}\left(\psi_{2}\right)\right]$. We substitute the definition of $H\left(\psi_{1}, \psi_{2}\right)$ from (3.4) on the right hand side.

$$
\psi_{1} \triangleright \psi_{2}=A-\left(A-\left(\psi_{1} \triangleright\left[\mathcal{M}\left(\psi_{2}\right)\right]\right)\right)-\mathcal{E}\left(\psi_{2}\right)
$$


When $X, Y$ and $\mathrm{Z}$ denote arbitrary sets, we have

$$
X-(Y-Z)=(X-Y) \cup(X \cap Y \cap Z),
$$

so

$$
\begin{aligned}
\psi_{1} \triangleright \psi_{2} & =(A-A) \cup\left(A \cap A \cap\left(\psi_{1} \triangleright\left[\mathcal{M}\left(\psi_{2}\right)\right]\right)\right)-\mathcal{E}\left(\psi_{2}\right) \\
& =\left(A \cap\left(\psi_{1} \triangleright\left[\mathcal{M}\left(\psi_{2}\right)\right]\right)\right)-\mathcal{E}\left(\psi_{2}\right) \\
& =\left(\psi_{1} \triangleright\left[\mathcal{M}\left(\psi_{2}\right)\right]\right)-\mathcal{E}\left(\psi_{2}\right) \\
& =\psi_{1} \triangleright\left(\left[\mathcal{M}\left(\psi_{2}\right)\right]-\mathcal{E}\left(\psi_{2}\right)\right) \\
& =\psi_{1} \triangleright \psi_{2}
\end{aligned}
$$

Lemma 3.10. $\psi_{2} \underline{e} \psi_{1}=\left(\left(\left[\mathcal{M}\left(\psi_{2}\right)\right] \stackrel{e}{[}\left[\mathcal{M}\left(\psi_{1}\right)\right]\right) \cup H\left(\psi_{1}, \psi_{2}\right)\right)-\mathcal{E}\left(\psi_{2}\right)$.

Proof. From Lemma 3.9 and equivalence (3.1) we have $\psi_{2} \underline{e} \psi_{1}=\psi_{2}-\left(\psi_{1} \triangleright \psi_{2}\right)$, or

$$
\psi_{2} \underline{e} \psi_{1}=\underbrace{\psi_{2}}_{A}-[(\underbrace{\left.\left[\mathcal{M}\left(\psi_{1}\right)\right] \triangleright\left[\mathcal{M}\left(\psi_{2}\right)\right]\right)}_{B}-\underbrace{H\left(\psi_{1}, \psi_{2}\right)}_{C})-\underbrace{\mathcal{E}\left(\psi_{2}\right)}_{D}]
$$

Using (3.5), we rewrite the right hand side equivalently

$$
\psi_{2} \underline{e} \psi_{1}=A-((B-C)-D)=(A-(B-C)) \cup(A \cap(B-C) \cap D)
$$

Since in our case $A \cap D=\emptyset$, therefore $(A \cap(B-C) \cap D)=\emptyset$ and we get

$$
\psi_{2} \stackrel{e}{s} \psi_{1}=(A-(B-C))=(A-B) \cup(A \cap B \cap C)
$$

We now evaluate $A \cap B \cap C$. We note that $A \cap B=\left(\left[\mathcal{M}\left(\psi_{2}\right)\right]-D\right) \cap B$ and since $(X-Y) \cap Z=$ $X \cap Z-Y \cap Z$, we have

$$
A \cap B=\left[\mathcal{M}\left(\psi_{2}\right)\right] \cap B-D \cap B=B-B \cap D=B-D .
$$

Next, $(B-D) \cap C=B \cap C-D \cap C$ and since $C \subseteq B,(B-D) \cap C=C-D \cap C=C-D$, so we get

$$
\begin{aligned}
\psi_{2} \frac{e}{\psi_{1}} \psi_{1} & =(A-B) \cup(C-D) \\
& =\left(\psi_{2}-\left(\left[\mathcal{M}\left(\psi_{1}\right)\right] \triangleright\left[\mathcal{M}\left(\psi_{2}\right)\right]\right)\right) \cup\left(H\left(\psi_{1}, \psi_{2}\right)-\mathcal{E}\left(\psi_{2}\right)\right) \\
& =\left(\left[\mathcal{M}\left(\psi_{2}\right)\right]-\mathcal{E}\left(\psi_{2}\right)-\left(\left[\mathcal{M}\left(\psi_{1}\right)\right] \triangleright\left[\mathcal{M}\left(\psi_{2}\right)\right]\right)\right) \cup\left(H\left(\psi_{1}, \psi_{2}\right)-\mathcal{E}\left(\psi_{2}\right)\right) \\
& =\left(\left[\mathcal{M}\left(\psi_{2}\right)\right] \underline{e}\left[\mathcal{M}\left(\psi_{1}\right)\right]-\mathcal{E}\left(\psi_{2}\right)\right) \cup\left(H\left(\psi_{1}, \psi_{2}\right)-\mathcal{E}\left(\psi_{2}\right)\right) \\
& =\left(\left(\left[\mathcal{M}\left(\psi_{2}\right)\right] \underline{e}\left[\mathcal{M}\left(\psi_{1}\right)\right]\right) \cup H\left(\psi_{1}, \psi_{2}\right)\right)-\mathcal{E}\left(\psi_{2}\right) .
\end{aligned}
$$

We calculate $\psi_{1} \triangleright \psi_{2}$ and $\psi_{1} \underline{e} \psi_{1}$ separately in each of the following three cases

Case 1: $M G U_{\subseteq}\left(\mathcal{M}\left(\psi_{1}\right), \mathcal{M}\left(\psi_{2}\right)\right)=\emptyset$, i.e. the image $\left[\mathcal{M}\left(\psi_{1}\right)\right] \triangleright\left[\mathcal{M}\left(\psi_{2}\right)\right]$ is empty.

Case 2: $M G U_{\subseteq}\left(\mathcal{M}\left(\psi_{1}\right), \mathcal{M}\left(\psi_{2}\right), \mathcal{V}\left(\psi_{1}\right)\right) \neq \emptyset$, i.e. by Theorem 3.3$]\left[\mathcal{M}\left(\psi_{1}\right)\right] \models\left[\mathcal{M}\left(\psi_{2}\right)\right]$ and hence the image $\left[\mathcal{M}\left(\psi_{1}\right)\right] \triangleright\left[\mathcal{M}\left(\psi_{2}\right)\right]$ equals the entire $\left[\mathcal{M}\left(\psi_{2}\right)\right]$.

Case 3: $M G U_{\subseteq}\left(\mathcal{M}\left(\psi_{1}\right), \mathcal{M}\left(\psi_{2}\right)\right) \neq \emptyset$, i.e. the image $\left[\mathcal{M}\left(\psi_{1}\right)\right] \triangleright\left[\mathcal{M}\left(\psi_{2}\right)\right]$ is non-empty.

Cases 1 and 3 are complementary. However we have separated case 2, which is a specific subcase of 3 , because it comes up while deciding $\psi$-form entailment (see Theorem [3.6). Moreover, case 3 is reduced to case 2, as we will demonstrate.

Case 1 is the simplest and is covered by Theorem 3.11 .

Theorem 3.11. If $M G U_{\subseteq}\left(\mathcal{M}\left(\psi_{1}\right), \mathcal{M}\left(\psi_{2}\right)\right)=\emptyset, \psi_{1} \triangleright \psi_{2}=\emptyset$ and $\psi_{2} \underline{e} \psi_{1}=\left\{\psi_{2}\right\}$. 
Proof. As follows from Theorem 3.7. $M G U_{\subseteq}\left(\mathcal{M}\left(\psi_{1}\right), \mathcal{M}\left(\psi_{2}\right)\right)=\emptyset$ implies that the image $\left[\mathcal{M}\left(\psi_{1}\right)\right] \triangleright\left[\mathcal{M}\left(\psi_{2}\right)\right]$ is empty. Since $\left(\psi_{1} \triangleright \psi_{2}\right) \subseteq\left(\left[\mathcal{M}\left(\psi_{1}\right)\right] \triangleright\left[\mathcal{M}\left(\psi_{2}\right)\right]\right)$, we have that $\psi_{1} \triangleright \psi_{2}=\emptyset$ and therefore by equivalence (B.1) $\psi_{2} \frac{e}{} \psi_{1}=\psi_{2}$.

Case 2 amounts to $\psi_{1}$ nearly entailing $\psi_{2}$.

Theorem 3.12. When $\left[\mathcal{M}\left(\psi_{1}\right)\right] \models\left[\mathcal{M}\left(\psi_{2}\right)\right]$

$$
\begin{gathered}
\psi_{1} \triangleright \psi_{2}=\psi_{2}-H\left(\psi_{1}, \psi_{2}\right) \\
\psi_{2} \frac{e}{\psi_{1}}=H\left(\psi_{1}, \psi_{2}\right)-\mathcal{E}\left(\psi_{2}\right)
\end{gathered}
$$

Proof. (3.6) and (3.7) trivially follow from Lemma 3.9 and definition (3.4) by substituting $\left[\mathcal{M}\left(\psi_{2}\right)\right]$ in place of $\left[\mathcal{M}\left(\psi_{1}\right)\right] \triangleright\left[\mathcal{M}\left(\psi_{2}\right)\right]$ and substituting $\emptyset$ in place of $\left[\mathcal{M}\left(\psi_{1}\right)\right] \underline{e}\left[\mathcal{M}\left(\psi_{2}\right)\right]$.

The expression for the set of holes $H\left(\psi_{1}, \psi_{2}\right)$ in this case is derived in Lemma 3.14. We first demonstrate the computation of the set of holes, image and e-difference in the following example.

The algorithm is straightforward when there is only one subset-unifier of $\mathcal{M}\left(\psi_{1}\right)$ with $\mathcal{M}\left(\psi_{2}\right)$, i.e. each clause of $\left[\mathcal{M}\left(\psi_{1}\right)\right] \triangleright\left[\mathcal{M}\left(\psi_{2}\right)\right]$ is entailed by exactly one clause of $\left[\mathcal{M}\left(\psi_{1}\right)\right]$. The set of holes in this case is simply the union of images from each exception of $\psi_{1}$ onto $\left[\mathcal{M}\left(\psi_{2}\right)\right]$.

Example 3.13. Consider

$$
\begin{gathered}
\psi_{1}=[\neg P(x, y, z) \text { except }\{\{x=B\},\{x=C, y=D\},\{x=A\}\}] \\
\psi_{2}=[\neg P(w, E, A) \text { except }\{\{w=G\}\}]
\end{gathered}
$$

Since there is only one subset-unifier of $\mathcal{M}\left(\psi_{1}\right)$ with $\mathcal{M}\left(\psi_{2}\right)$, the image of $\psi_{1}$ onto $\psi_{2}$ is simply the image $\left[\mathcal{M}\left(\psi_{1}\right)\right]$ onto $\left[\mathcal{M}\left(\psi_{2}\right)\right]$ minus exceptions of $\psi_{2}$ and the image of exceptions of $\psi_{1}$ on $\left[\mathcal{M}\left(\psi_{2}\right)\right]$, i.e.

$$
\psi_{1} \triangleright \psi_{2}=\left[\mathcal{M}\left(\psi_{1}\right)\right] \triangleright\left[\mathcal{M}\left(\psi_{2}\right)\right]-\mathcal{E}\left(\psi_{2}\right)-\bigcup_{i=1}^{3}\left(\left[\mathcal{E}_{i}\left(\psi_{1}\right)\right] \triangleright\left[\mathcal{M}\left(\psi_{2}\right)\right]\right)
$$

In this case $\left[\mathcal{M}\left(\psi_{1}\right)\right] \triangleright\left[\mathcal{M}\left(\psi_{2}\right)\right]=\left[\mathcal{M}\left(\psi_{2}\right)\right]$ and, since (by definition (2.3)) $\psi_{2}=$ $\left[\mathcal{M}\left(\psi_{2}\right)\right]-\mathcal{E}\left(\psi_{2}\right)$

$$
\begin{aligned}
\psi_{1} \triangleright \psi_{2}= & {[\neg P(w, E, A) \text { except }\{\{w=G\}\}]-[\neg P(B, y, z)] \triangleright[\neg P(w, E, A)] } \\
& -[\neg P(C, D, z)] \triangleright[\neg P(w, E, A)][\neg P(A, y, z)] \triangleright[\neg P(w, E, A)] \\
= & {[\neg P(w, E, A) \text { except }\{\{w=G\}\}]-[\neg P(B, E, A)]-[\neg P(A, E, A)] } \\
= & {[\neg P(w, E, A) \text { except }\{\{w=G\},\{w=B\},\{w=A\}\}] }
\end{aligned}
$$

Computing the holes is more complex when there is more than one subset-unifier of $\mathcal{M}\left(\psi_{1}\right)$ with $\mathcal{M}\left(\psi_{2}\right)$, because in this case some clauses of $\left[\mathcal{M}\left(\psi_{1}\right)\right] \triangleright\left[\mathcal{M}\left(\psi_{2}\right)\right]$ are entailed by more than one clause of $\left[\mathcal{M}\left(\psi_{1}\right)\right]$. Then, even though an exception removes from $\left[\mathcal{M}\left(\psi_{1}\right)\right]$ an entailing clause for some clause $c$ of $\psi_{2}, c$ may be entailed by another clause in $\psi_{1}$, and consequently the set of holes is not simply a set of images from $\psi_{1}$ 's exceptions, but rather an intersection of such images. Example 3.15illustrates this computation.

We derive an expression for calculating $H\left(\psi_{1}, \psi_{2}\right)$ in the next Lemma by first introducing the set of $\psi$-forms within $\left[\mathcal{M}\left(\psi_{1}\right)\right]$ (denoted $\Psi_{1}$ ), all of which entail some part of $\left[\mathcal{M}\left(\psi_{2}\right)\right]$, and showing how to combine them to calculate $H\left(\psi_{1}, \psi_{2}\right)$. 


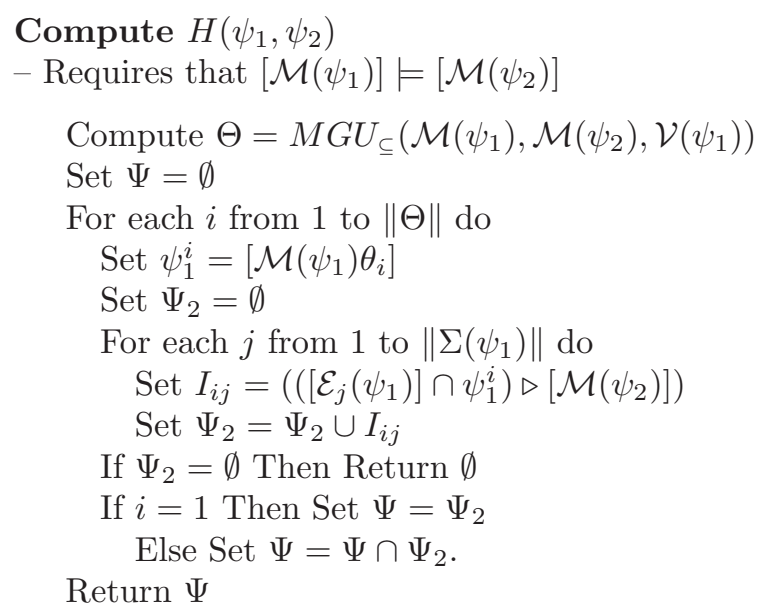

ComputeImg2 $\left(\psi_{1}, \psi_{2}\right)$

- Requires that $\left[\mathcal{M}\left(\psi_{1}\right)\right] \models\left[\mathcal{M}\left(\psi_{2}\right)\right]$

Set $\Psi_{H}=$ ComputeH $\left(\psi_{1}, \psi_{2}\right), \psi=\psi_{2}$

For each simple $\psi$-form $\psi_{h} \in \Psi_{H}$

Set $\psi=\psi-\psi_{h}$

Return $\{\psi\}$

\section{ComputeEDiff2 $\left(\psi_{2}, \psi_{1}\right)$}

- Requires that $\left[\mathcal{M}\left(\psi_{1}\right)\right] \models\left[\mathcal{M}\left(\psi_{2}\right)\right]$

Set $\Psi_{H}=$ Compute $H\left(\psi_{1}, \psi_{2}\right), \Psi=\emptyset$

For each simple $\psi$-form $\psi_{h} \in \Psi_{H}$

Set $\psi=\psi_{h}$

For each simple $\psi$-form $\psi_{e} \in \mathcal{E}(\psi)$

Set $\psi=\psi-\psi_{e}$

Set $\Psi=\Psi \cup\{\psi\}$

Return $\Psi$

Figure 9: Computing the set of holes, image and e-difference operations in case $\psi_{1}$ nearly entails $\psi_{2}$.

Lemma 3.14. Let $\Theta=M G U_{\subseteq}\left(\mathcal{M}\left(\psi_{1}\right), \mathcal{M}\left(\psi_{2}\right), \mathcal{V}\left(\psi_{1}\right)\right)$ be nonempty. Let $\Psi_{1}$ be defined as follows.

$$
\Psi_{1}=\left\{\psi_{1}^{i} \mid \psi_{1}^{i}=\left[\mathcal{M}\left(\psi_{1}\right) \theta_{i}\right], \theta_{i} \in \Theta, 1 \leq i \leq\|\Theta\|\right\}
$$

Then,

$$
H\left(\psi_{1}, \psi_{2}\right)=\bigcap_{\psi_{1}^{i} \in \Psi_{1}} \bigcup_{j=0}^{\left\|\Sigma\left(\psi_{1}\right)\right\|}\left(\left(\left[\mathcal{E}_{j}\left(\psi_{1}\right)\right] \cap \psi_{1}^{i}\right) \triangleright\left[\mathcal{M}\left(\psi_{2}\right)\right]\right),
$$

Proof. Each $\psi_{1}^{i}$ entails $\left[\mathcal{M}\left(\psi_{2}\right)\right]$, i.e. $\psi_{1}^{i} \triangleright\left[\mathcal{M}\left(\psi_{2}\right)\right]=\left[\mathcal{M}\left(\psi_{2}\right)\right]$ for each $1 \leq i \leq\|\Theta\|$, because the main clause of each $\psi_{1}^{i}$ equals some subset of literals of $\mathcal{M}\left(\psi_{2}\right)$. However, each $\psi_{1}^{i}$ may contain clauses that are exceptions of $\psi_{1}$, namely $\bigcup_{j=0}^{\|\Sigma\|\left(\psi_{1}\right)}\left(\left[\mathcal{E}_{j}\left(\psi_{1}\right)\right] \cap \psi_{1}^{i}\right)$. We call these clauses $\psi_{1}$ 's exceptions in $\psi_{1}^{i}$.

Thus, $\phi\left(\Psi_{1}\right)$ contains all clauses of $\psi_{1}$ that entail something in $\left[\mathcal{M}\left(\psi_{2}\right)\right]$ and more, namely $\psi_{1}$ 's exceptions in $\psi_{1}^{i}$. Therefore

$$
\psi_{1} \triangleright\left[\mathcal{M}\left(\psi_{2}\right)\right]=\bigcup_{\psi_{1}^{i} \in \Psi_{1}}\left(\left(\psi_{1}^{i}-\cup_{j=0}^{\left\|\Sigma\left(\psi_{1}\right)\right\|}\left(\left[\mathcal{E}_{j}\left(\psi_{1}\right)\right] \cap \psi_{1}^{i}\right)\right) \triangleright\left[\mathcal{M}\left(\psi_{2}\right)\right]\right) .
$$

Note that for a given $\psi_{1}^{i} \in \Psi_{1}$, each clause of $\left[\mathcal{M}\left(\psi_{2}\right)\right]$ is entailed by exactly one clause of $\psi_{1}^{i}$, i.e. for every $\psi_{1}^{i} \in \Psi_{1}$, and every $c \in\left[\mathcal{M}\left(\psi_{2}\right)\right],\left(\psi_{1}^{i} \models c\right) \Leftrightarrow \exists ! c_{1} \in \psi_{1}^{i} \cdot c_{1} \models c$ (existence of a clause $\mathcal{M}\left(\psi_{2}\right) \sigma$ such that there are 2 different clauses $c^{\prime}, c^{\prime \prime} \in \psi_{1}^{i}$ that entail it, leads to a contradiction to the fact that $\psi_{2}$ and $\psi_{1}$ are fixed length $\psi$-forms). The last observation 
allows to distribute the image operator and rewrite the last expression as follows

$$
\begin{aligned}
\psi_{1} \triangleright\left[\mathcal{M}\left(\psi_{2}\right)\right] & =\bigcup_{\psi_{1}^{i} \in \Psi_{1}}\left(\left(\psi_{1}^{i} \triangleright\left[\mathcal{M}\left(\psi_{2}\right)\right]\right)-\bigcup_{j=0}^{\left\|\Sigma\left(\psi_{1}\right)\right\|}\left(\left[\mathcal{E}_{j}\left(\psi_{1}\right)\right] \cap \psi_{1}^{i}\right) \triangleright\left[\mathcal{M}\left(\psi_{2}\right)\right]\right) \\
& =\left[\mathcal{M}\left(\psi_{2}\right)\right]-\bigcap_{\psi_{1}^{i} \in \Psi_{1}} \bigcup_{j=0}^{\left\|\Sigma\left(\psi_{1}\right)\right\|}\left(\left(\left[\mathcal{E}_{j}\left(\psi_{1}\right)\right] \cap \psi_{1}^{i}\right) \triangleright\left[\mathcal{M}\left(\psi_{2}\right)\right]\right) .
\end{aligned}
$$

i.e. the set of clauses of $\left[\mathcal{M}\left(\psi_{2}\right)\right]$ not entailed by $\psi_{1}$ is the intersection of images of $\psi_{1}$ 's exceptions in all of $\psi_{1}^{i} \in \Psi_{1}$,

The formula for $H\left(\psi_{1}, \psi_{2}\right)$ follows from substituting the derived expression for $\psi_{1} \triangleright$ $\left[\mathcal{M}\left(\psi_{2}\right)\right]$ in the definition (3.4) and noticing that since $\psi_{1}$ nearly entails $\psi_{2},\left[\mathcal{M}\left(\psi_{1}\right)\right] \triangleright$ $\left[\mathcal{M}\left(\psi_{2}\right)\right]=\left[\mathcal{M}\left(\psi_{2}\right)\right]$.

The procedure for computing the set of holes $H\left(\psi_{1}, \psi_{2}\right)$ in case $\psi_{1}$ nearly entails $\psi_{2}$ presented in Figure 9 is based on Lemma 3.14. Procedures for computing the image and e-difference in case $\psi_{1}$ nearly entails $\psi_{2}$ are also presented in Figure 9 .

Example 3.15. Consider

$$
\begin{aligned}
& \psi_{1}=[\neg P(x, y, z) \text { except }\{\{x=B\},\{x=C, y=D\},\{x=A\}\}] \\
& \psi_{2}=[\neg P(w, E, A) \vee \neg P(C, D, w) \vee \neg Q(w) \text { except }\{\{w=G\}\}]
\end{aligned}
$$

Here, the clause $c=\neg P(K, E, A) \vee \neg P(C, D, K) \vee \neg Q(K)$, for example, is entailed by two clauses of $\left[\mathcal{M}\left(\psi_{1}\right)\right]$, namely, by $\neg P(K, E, A)$ and $\neg P(C, D, K)$. Although the second of these clauses is not in $\psi_{1}$ due to the second exception, the first one, $\neg P(K, E, A)$ is in $\psi_{1}$ and therefore $\psi_{1} \models \neg P(K, E, A) \vee \neg P(C, D, K) \vee \neg Q(K)$. Thus, even though $c \in\left[\mathcal{E}_{2}\left(\psi_{1}\right)\right] \triangleright \psi_{2}$, $c \in \psi_{1} \triangleright \psi_{2}$.

Computing the set of holes according to Lemma 3.14 yields

$$
\begin{aligned}
& H\left(\psi_{1}, \psi_{2}\right)=\left(\left(\mathcal{E}\left(\psi_{1}\right) \cap \psi_{1}^{1}\right) \triangleright\left[\mathcal{M}\left(\psi_{2}\right)\right]\right) \cap\left(\left(\mathcal{E}\left(\psi_{1}\right) \cap \psi_{1}^{2}\right) \triangleright\left[\mathcal{M}\left(\psi_{2}\right)\right]\right)= \\
&\left\{\begin{array}{c}
{[\neg P(B, E, A) \vee \neg P(C, D, B) \vee \neg Q(B)],} \\
{[\neg P(A, E, A) \vee \neg P(C, D, A) \vee \neg Q(A)]}
\end{array}\right\} .
\end{aligned}
$$

Furthermore, according to Theorem 3.12 e-difference $\psi_{2} \underline{e} \psi_{1}$ equals the set of holes $H\left(\psi_{1}, \psi_{2}\right)$ minus exceptions of $\psi_{2}$

$$
\begin{aligned}
\psi_{2} \underline{e} \psi_{1}= & H\left(\psi_{1}, \psi_{2}\right)-\{[\neg P(G, E, A) \vee \neg P(C, D, G) \vee \neg Q(G)]\}= \\
& \left\{\begin{array}{c}
{[\neg P(B, E, A) \vee \neg P(C, D, B) \vee \neg Q(B)],} \\
{[\neg P(A, E, A) \vee \neg P(C, D, A) \vee \neg Q(A)]}
\end{array}\right\} .
\end{aligned}
$$

The image $\psi_{1} \triangleright \psi_{2}$ equals $\left[\mathcal{M}\left(\psi_{2}\right)\right]$ minus the set of holes, and minus exceptions of $\psi_{2}$, i.e.

$$
\begin{aligned}
\psi_{1} \triangleright \psi_{2} & =\psi_{2}-H\left(\psi_{1}, \psi_{2}\right) \\
& =[\neg P(w, E, A) \vee \neg P(C, D, w) \vee \neg Q(w) \text { except }\{\{w=G\}\}]-H\left(\psi_{1}, \psi_{2}\right) \\
& =[\neg P(w, E, A) \vee \neg P(C, D, w) \vee \neg Q(w) \text { except }\{\{w=G\},\{w=A\},\{w=B\}\}]
\end{aligned}
$$

Recall that the computation of e-difference in case $\left[\mathcal{M}\left(\psi_{1}\right)\right] \models\left[\mathcal{M}\left(\psi_{2}\right)\right]$ comes up in verifying entailment (Theorem 3.6). The following Observation guarantees that each $\psi$-form in the e-difference $\psi_{2} \underline{e} \psi_{1}$ is strictly "smaller" than $\psi_{2}$. This observation plays a critical role in establishing the complexity bounds on $\psi$-form reasoning. 
Observation 3.16. Assume $\left[\mathcal{M}\left(\psi_{1}\right)\right] \models\left[\mathcal{M}\left(\psi_{2}\right)\right]$ and assume that none of the exception forms of $\psi_{1}$ entails $\left[\mathcal{M}\left(\psi_{2}\right)\right]$. Then each $\psi$-form in $\psi_{2} \underline{e}-\psi_{1}$ uses strictly fewer variables than there are in $\psi_{2}$.

Proof. As follows from Theorem 3.12 and Lemma 3.14 the e-difference is a subset of a union of images of exceptions of $\psi_{1}$ on $\left[\mathcal{M}\left(\psi_{2}\right)\right]$. Each such image is obtained by instantiating the main clause $\mathcal{M}\left(\psi_{2}\right)$ with a subset unifying substitution, call it $\sigma$. When $\sigma$ does not bind any variables of $\psi_{2}$ to constants, the image is equal to $\left[\mathcal{M}\left(\psi_{2}\right)\right]$, which contradicts the conditions of the Observation. Thus, $\sigma$ must bind some variables of $\mathcal{M}\left(\psi_{2}\right)$ to constants, and therefore $\left[\mathcal{M}\left(\psi_{2}\right) \sigma\right]$, and in turn, every subset of this $\psi$-form is expressed with a $\psi$-form that contains strictly fewer variables than $\left[\mathcal{M}\left(\psi_{2}\right)\right]$.

We now consider case 3. There is a non-empty image of the main part of $\psi_{1}$ onto the main part of $\psi_{2}$ which occurs when $M G U_{\subseteq}\left(\mathcal{M}\left(\psi_{1}\right), \mathcal{M}\left(\psi_{2}\right)\right) \neq \emptyset$. In this case we first compute the image $\left[\mathcal{M}\left(\psi_{1}\right)\right] \triangleright\left[\mathcal{M}\left(\psi_{2}\right)\right]$, denoted below by $\Psi$. Every $\psi$-form in $\Psi$ is nearly entailed by $\psi_{1}$, and thus we can compute the image of $\psi_{1}$ on each of $\psi$-forms in $\Psi$ using the methods of Case 2. The image $\psi_{1} \triangleright \psi_{2}$ equals the union of images of $\psi_{1}$ onto each $\psi$-form in $\Psi$, minus exceptions of $\psi_{2}$.

Theorem 3.17. Let $M G U_{\subseteq}\left(\mathcal{M}\left(\psi_{1}\right), \mathcal{M}\left(\psi_{2}\right)\right) \neq \emptyset$, and let $\Psi$ denote the image $\left[\mathcal{M}\left(\psi_{1}\right)\right] \triangleright$ $\left[\mathcal{M}\left(\psi_{2}\right)\right]$. Then

$$
\begin{gathered}
\psi_{1} \triangleright \psi_{2}=\left(\psi_{1} \triangleright \Psi\right)-\mathcal{E}\left(\psi_{2}\right) \\
\psi_{2} \stackrel{e}{\psi_{1}}=\left(\psi_{2}-\Psi\right) \cup\left[\left(\Psi-\mathcal{E}\left(\psi_{2}\right)\right) \frac{e}{} \psi_{1}\right]
\end{gathered}
$$

Proof. By Theorem $3.7 M G U_{\subseteq}\left(\mathcal{M}\left(\psi_{1}\right), \mathcal{M}\left(\psi_{2}\right)\right) \neq \emptyset$ implies that the image $\Psi$ is non-empty and thus consists of a set of simple $\psi$-forms. Each $\psi$-form in $\Psi$ is nearly entailed by $\psi_{1}$. The image $\psi_{1} \triangleright \psi_{2}$ is a subset of $\Psi$, and equals exactly the set of all clauses in $\Psi$ that are not exceptions of $\psi_{2}$ and that are entailed by $\psi_{1}$, i.e.

$$
\psi_{1} \triangleright \psi_{2}=\left(\psi_{1} \triangleright \Psi\right)-\mathcal{E}\left(\psi_{2}\right) .
$$

Since each of $\psi$-forms in $\Psi$ is nearly entailed by $\psi_{1}$ the calculation of the image $\psi_{1} \triangleright \Psi$ in the above expression can be carried out according to Theorem 3.12

The proof of (3.11) is similar. The part of $\psi_{2}$ that is not entailed by $\psi_{1}$ includes $\psi_{2}-\Psi$ plus parts of $\Psi$ that are not exceptions of $\psi_{2}$ and are not entailed by $\psi_{1}$, i.e. $\left(\Psi-\mathcal{E}\left(\psi_{2}\right)\right) \underline{e} \psi_{1}$.

The procedures for computing image and e-difference in case 3 are given in Figure 10.

Theorem 3.18. Image and e-difference of two fixed length $\psi$-forms is equivalent to a finite set of fixed length $\psi$-forms.

Proof. The fact that all operations produce sets of $\psi$-forms follows from the fact that all of them produce subsets of operand $\psi$-forms. The fact that indeed this set is finite follows from the Theorems 3.113 .17

The resulting $\psi$-forms are fixed length, because they contain clauses from argument $\psi$ forms, and each subset $\psi$-form of a fixed length $\psi$-form is obviously a fixed length $\psi$-form. 

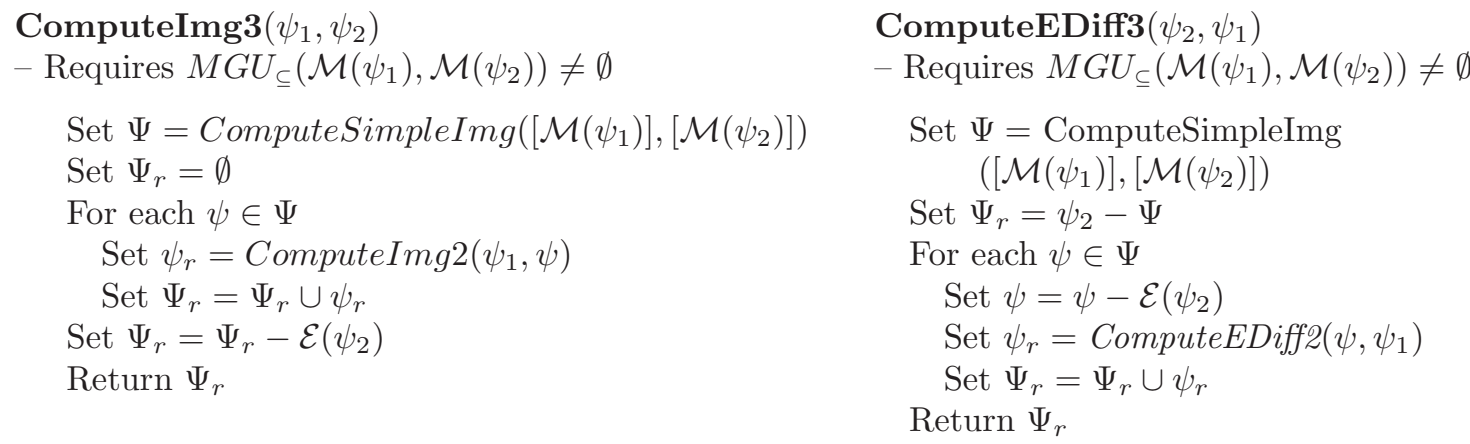

Figure 10: Procedures ComputeImg3 $\left(\psi_{1}, \psi_{2}\right)$ and ComputeEDiff3 $\left(\psi_{1}, \psi_{2}\right)$ compute $\psi_{1} \triangleright \psi_{2}$ and $\psi_{2} \frac{e}{} \psi_{1}$ in case there is a non-empty image of the main part of $\psi_{1}$ onto the main part of $\psi_{2}$, i.e. $\left[\mathcal{M}\left(\psi_{1}\right)\right] \triangleright\left[\mathcal{M}\left(\psi_{2}\right)\right] \neq \emptyset$

3.6. Complexity of $\psi$-form operations. The recursive procedure for determining entailment $\Psi=\psi$ based on Theorem 3.6] takes time $O(n)$, where $n$ is the number of $\psi$-forms in $\Psi$, when the maximum number of exceptions, and variables and literals in the main clause of a $\psi$-form are fixed. We assume unification takes constant bounded time, which is guaranteed when the cardinality of predicate symbols is bounded by a constant. These assumptions are common in open world applications:

- the number of variables and literals in the main clause and cardinality of predicate symbols are always finite and bounded by the specification of the initial and goal states and the action descriptions. Moreover, they are typically small.

- the number of exceptions is limited by a function of the number of objects known in the initial state and those objects created by the actions in a constructed plan. When the length of the plan is constant bounded, the number of exceptions is therefore also constant bounded. In general, the complexity of entailment is polynomially bounded in the maximum number of exceptions, as presented in Figure [1] and discussed briefly at the end of this section.

To obtain the linear bound on the complexity of entailment, notice that finding a $\psi$ form that nearly entails $\psi$ requires a pass through at most $n \psi$-forms of $\Psi$ spending constant time at each, since checking nearly entailment takes constant time. Once a nearly entailing $\psi$-form $\psi_{k}$ is found, we calculate the difference $\psi \underline{e} \psi_{k}$ and apply Theorem (3.6) to each $\psi$-form in the e-difference. This is a recursive procedure, which can be represented by a recursion tree. In the tree, each node represents the non-recursive computation, i.e. finding a nearly entailing $\psi$-form $\psi_{k}$, and computing the e-difference $\psi \underline{\underline{e}} \psi_{k}$; and each branch represents a recursive call to the same procedure for checking entailment of each $\psi$-form in the e-difference $\psi \underline{e} \psi_{k}$. The complexity of the entire procedure equals the sum of the complexities at the nodes of the recursion tree. The time spent at each node is proportional to the number of $\psi$-forms in $\Psi$, which is $n$ at the root of the tree, and decreases by one at each subsequent level. The branching factor $\beta$ at each node equals the number of $\psi$-forms in the e-difference $\psi \underline{e} \psi_{k}$. $\beta$ is constant bounded when we bound by constants the maximum number of exceptions, variables and literals in the $\psi$-forms. Therefore, at each level $i$ of 
the tree we have at most $\beta^{i}$ nodes, and computation at each node has time complexity proportional to $(n-i)$.

The depth of the recursion tree is bounded by $n$. However, it is also bounded by $\min (n, V+1)$, where $V$ is the maximum number of variables in a $\psi$-form. As follows from Observation 3.16 (page 221) unless $\psi \underline{e} \psi_{k}=\{\psi\}$, each $\psi$-form in the difference $\psi \underline{e} \psi_{k}$ uses strictly fewer variables than the original $\psi$, because when $\psi_{k}$ nearly entails $\psi$, all $\psi$-forms in the e-difference $\psi \underline{e} \psi_{k}$ are subsets of images from $\psi_{k}$ 's exceptions, and unless an exception entails the whole $\psi$, this image is obtained by instantiating some variables of $\psi$. In the case where $\psi \underline{e} \psi_{k}=\{\psi\}$, the branching factor out of the node equals one, and we can collapse the parent and the child into one node. Thus, assuming $V$ is less than $n$, the depth of recursion in checking $\Psi \models \psi$ is bounded by the maximum number of variables in a $\psi$-form, $V$. The overall time complexity bound is $O\left(\beta^{V} n\right)=O(n)$, since $\beta$ and $V$ are constants.

Figure 11] shows time complexity bounds of the $\psi$-form calculus operations as functions of the number of participating $\psi$-forms $n$, maximum number of exceptions $E$, maximum number of variables $V$, and maximum number of literals in a $\psi$-form clause $C$. The complete treatment of the time complexity issues of the calculus of $\psi$-forms can be found in [2].

$$
\left\{\psi_{1}, \ldots, \psi_{n}\right\} \models \psi \quad \psi_{1} \triangleright \psi_{2} \quad \psi_{2} \frac{e}{\psi_{1}}
$$

\begin{tabular}{cccc}
\hline Simple fixed length $\psi$-forms & $O(n)$ & $O(1)$ & $O(1)$ \\
Non-simple fixed length $\psi$-forms & $O\left(n E^{V(t+1)+1}\right)$ & $O\left(E^{t}\right)$ & $O\left(E^{t+1}\right)$ \\
Non-simple limited form $\psi$-forms & $O\left(n E^{V+1}\right)$ & $O(E)$ & $O\left(E^{2}\right)$ \\
Singleton $\psi_{1}$ & $O(n E)$ & $O(E)$ & $O(E)$
\end{tabular}

Figure 11: Time complexity of computing $\psi$-form operations. Assumes unification takes constant time and the maximum number of literals $(C)$ and variables $(V)$ in the main form of a $\psi$-form are constant. $E$ denotes the maximum number of exceptions, $t$ denotes the maximum number of possible subset matches between main forms of two $\psi$-forms. $t=O\left(e^{C / e}\right)$, where $e$ is the Euler's number, for fixed length $\psi$-forms.

To summarize: $\psi$-form entailment takes linear time in the number of participating $\psi$-forms $n$, when the maximum length of clause, maximum number of variables in a $\psi$ form and maximum number of exceptions are all fixed. When the number of exceptions is proportional to $n$, computing entailment remains bounded by a polynomial of the order proportional to the maximum number of variables in a $\psi$-form times the number of possible subset-matches between the main clauses. The complexity of $\psi$-form operations depends on the number of subset-matches between the main clauses of two $\psi$-forms, which in case of unrestricted $\psi$-forms is $C^{C}$. In fixed length $\psi$-forms the number of subset-matches between the main forms of two $\psi$-forms is bounded by $e^{C / e}$, as stated by the next Observation. To limit the number of subset-matches to at most one, $\psi$-forms can be restricted to contain no duplicate occurrences of a predicate symbol in the main clause. We call such $\psi$-forms limited form $\psi$-forms. 
Lemma 3.19. Let $\psi_{1}$ and $\psi_{2}$ be fixed length $\psi$-forms. In two different subset-matches of $\mathcal{M}\left(\psi_{1}\right)$ onto $\mathcal{M}\left(\psi_{2}\right)$, no two different literals of $\mathcal{M}\left(\psi_{1}\right)$ match the same literal of $\mathcal{M}\left(\psi_{2}\right)$.

Proof. As always, we assume that there is no overlap between the variables in two different $\psi$-forms. Suppose that the above statement is not true, i.e. there exist two different subsetmatches of $\mathcal{M}\left(\psi_{1}\right)$ onto $\mathcal{M}\left(\psi_{2}\right)$ that match two different literals $d_{1}$ and $d_{2}$ of $\mathcal{M}\left(\psi_{1}\right)$ on the same literal $d$ of $\mathcal{M}\left(\psi_{2}\right)$. Let $\sigma_{1}$ and $\sigma_{2}$ be substitutions corresponding to each subset-match, i.e. $\sigma_{1}$ and $\sigma_{2}$ are in $M G U_{\subseteq}\left(\mathcal{M}\left(\psi_{1}\right), \mathcal{M}\left(\psi_{2}\right), \mathcal{V}\left(\psi_{1}\right)\right)$.

Consider the following substitution $\sigma$.

- If $d_{1}$ and $d_{2}$ do not share variables, construct $\sigma$ as follows: combine bindings on variables in $d_{1}$ from $\sigma_{1}$ and bindings on variables in $d_{2}$ from $\sigma_{2}$. Then, obviously, $d_{1} \sigma=d_{2} \sigma=d$.

- Otherwise, if $d_{1}$ and $d_{2}$ do share variables, rename variables in $d_{2}$ so that there is no overlap with variables in $d_{1}$. Modify $\sigma_{2}$ by renaming the variables in the same way we did with $d_{2}$, and construct $\sigma$ as in the previous case. Again, $d_{1} \sigma=d_{2} \sigma$.

Thus, both cases produced a contradiction to the fact that no two literals of a fixed length $\psi_{1}$ unify.

Observation 3.20. Let $\psi_{1}$ and $\psi_{2}$ be fixed length $\psi$-forms. The number of subset-matches of $\mathcal{M}\left(\psi_{1}\right)$ onto $\mathcal{M}\left(\psi_{2}\right)$ is bounded by $e^{C / e}$, i.e. $\left\|M G U_{\subseteq}\left(\mathcal{M}\left(\psi_{1}\right), \mathcal{M}\left(\psi_{2}\right), \mathcal{V}\left(\psi_{1}\right)\right)\right\| \leq\left(e^{C / e}\right)$.

Proof. Suppose there are $i_{k}$ literals in $\mathcal{M}\left(\psi_{2}\right)$ that matched the k-th literal of $\mathcal{M}\left(\psi_{1}\right)$. Since according to Lemma 3.19 (page 24) no two different matches can match two different literals of $\mathcal{M}\left(\psi_{1}\right)$ to the same literal of $\mathcal{M}\left(\psi_{2}\right), i_{1}+\ldots+i_{k} \leq C$, otherwise two literals of $\mathcal{M}\left(\psi_{1}\right)$ would match the same literal of $\mathcal{M}\left(\psi_{2}\right)$.

The maximum size of $M G U_{\subseteq}\left(\mathcal{M}\left(\psi_{1}\right), \mathcal{M}\left(\psi_{2}\right), \mathcal{V}\left(\psi_{1}\right)\right)$ is bound by the product $i_{1} \times \ldots \times$ $i_{k}$. By the Cauchy's inequality

$$
\frac{i_{1}+\ldots+i_{k}}{k} \geq\left(i_{1} \times \ldots \times i_{k}\right)^{1 / k}
$$

The product $\left(i_{1} \times \ldots \times i_{k}\right)$ is limited by $\left(\frac{C}{k}\right)^{k}$ with the equality reachable only when $i_{1}=$ $i_{2}=\ldots=i_{k}=\frac{C}{k}$. Then the product $i_{1} \times \ldots \times i_{k}$. The maximum of this product is reached when $\log \left(\frac{C}{k}\right)-1=0$, i.e. when $\frac{C}{k}=e$, and equals $e^{C / e}$.

Thus, when the maximum number of disjuncts in a $\psi$-form is $C$, number of possible subset-matches is bounded by $e^{C / e}$.

\section{PSIPLAN REPRESENTATION}

As in previous work on open world planning, we assume that the world evolves as a sequence of states, where the transitions occur only as the result of deliberate action taken by the single agent. Since the agent's model of the world is incomplete, the actual state of the world differs from the state of the agent's knowledge of the world, which we call SOK. The agent's knowledge of the world is assumed to be correct. 
4.1. States of Knowledge. PSIPLAN propositions. A SOK is a set of propositions that represents what the agent knows is true about the world. In PSIPLAN, a SOK is a finite set of PSIPLAN domain propositions or, simply, propositions, which are defined to be either ground atoms or $\psi$-forms.

Since the agent's theory of the world is assumed to be correct, every proposition that a SOK entails is true in the actual world. Moreover, we make the following closed knowledge assumption (CKA): A literal $L$ is known to be true in $s$ if $s \models L$, known to be false if $s \models \neg L$ and unknown if both $s \not \models L$ and $s \not \forall \neg L$. This assumption is closed because entailment is decidable in PSIPLAN.

A model of a PSIPLAN proposition is a world in which it is true. We refer to the set of all models of a SOK $s$, denoted $I(s)$, as the set of possible worlds of $s$. It is the set of all worlds in which everything known by the agent is true. Correctness of the agents SOK implies that the set of models of a SOK always contains the actual world.

We use symbols $w, w^{\prime}, w_{1} \ldots w_{n}$ to refer to worlds, $W, W^{\prime}$ to refer to the sets of worlds, and $s, s^{\prime}, s_{1} \ldots s_{n}$ to refer to the agent's SOK. $\mathcal{W}$ denotes a set of all worlds.

4.2. Entailment in PSIPLAN. First observe that any consistent set $s$ of atoms plus $\psi$-forms does not entail any atoms but those in $s$. However, it may entail more $\psi$-forms than are entailed by $\psi$-forms of $s$ alone, because of the possibility of resolution between a ground clause $c$, represented by a $\psi$-form, and some atom $a$, such that $\neg a$ is a literal of $c$. However, once all resolutions are performed and the resolvents added to $s$, each $\psi$-form entailed by $s$ is entailed by the set of only $\psi$-forms of $s$, and each atom entailed by $s$ is in $s$. In other words, $s$ becomes saturated, i.e. for any ground proposition $q$ entailed by $s$ there is a single proposition $p \in s$ that entails $q$ (see also Theorem 3.2). A set of propositions $s$ is saturated if and only if for any ground proposition $q$,

$$
(s \models q) \Longrightarrow \exists p .(p \in s) \wedge(p \models q) .
$$

Thus, when $s$ is saturated, one need not combine elements of $s$ (through resolution) in order to show entailment. To determine entailment of an atom, $s \models a, a$ must be found in $s$. Entailment of any ground negated clause, or, in other words, singleton $\psi, s \models \psi$ is completely determined by entailment from a single $\psi$-form in $s$.

A saturated equivalent of $s$ is obtained by computing all possible resolutions from the unit clause resolution rule $\frac{a, \neg a \vee \neg a_{1} \vee \ldots \vee \neg a_{n}}{\neg a_{1} \vee \ldots \vee \neg a_{n}}$ between domain atoms in $s$ and clauses represented by $\psi$-forms.

For example, suppose

$$
s=\{\operatorname{In}(\text { paper }, / \text { tex }), \psi=[\neg \operatorname{In}(x, y) \vee \neg T(x, P S) \text { except }\{\{y=/ i m g\}\}]\} .
$$

Here, $\operatorname{In}(x, y)$ states that file $x$ is in directory $y$, and $T(x, P S)$ states that file $x$ has type Postscript.

$s$ is not saturated because, even though it entails that file paper is not a Postscript file, no single proposition of $s$ alone entails $\neg T$ (paper, $P S)$.

However, $\psi$ in $s$ contains a clause $c=\neg I n($ paper,$/$ tex $) \vee \neg T($ paper, $P S)$, and we can perform a resolution between $c$ and the atom In(paper, /tex), resulting in $\neg T($ paper, $P S)$. We add the $\psi$-form $[\neg T($ paper, $P S)]$ to the initial SOK, $s_{0}$.

The procedure Saturate $(s)$, depicted in Figure 12, returns a saturated equivalent of $s$ and consists of the following steps. Initially we set $s_{0}=s$. For every $\psi$-form $\psi$ in $s_{0}$ and for 
every atom $a$, we compute $(\neg a) \triangleright \psi$, as those are all and only clauses for which resolution is possible. If this image is empty, we go to the next $\psi$-form in $s_{0}$. Otherwise, suppose $(\neg a) \triangleright \psi=\psi^{\prime}$. From the properties of the image, it follows that $\neg a$ is a subclause of $\mathcal{M}\left(\psi^{\prime}\right)$. Let $\psi_{\text {new }}$ denote the $\psi$-form that is obtained from $\psi^{\prime}$ by removing $\neg a$ from its main clause, i.e. $\psi_{\text {new }}=\left[\mathcal{M}\left(\psi^{\prime}\right)-(\neg a)\right.$ except $\left.\Sigma\left(\psi^{\prime}\right)\right]$. We add $\psi_{\text {new }}$ to $s_{0}$, and continue until all $\psi$-forms in $s_{0}$, including the newly added, are processed in this way.

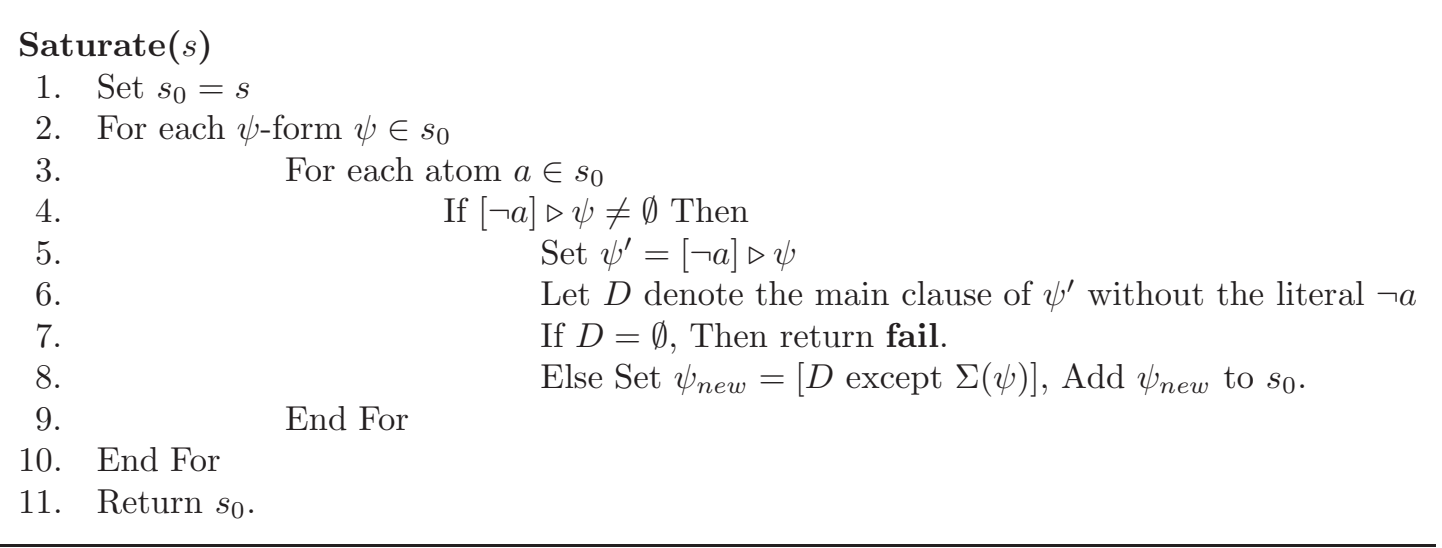

Figure 12: Procedure Saturate $(s)$. Preprocessing the Initial SOK. If set $s$ is unsatisfiable, returns fail.

Notice that, as a side effect, procedure Saturate() determines if $s$ is consistent. If at any moment we obtain an empty clause as a main part of some $\psi_{\text {new }}$, that indicates that $\mathcal{M}\left(\psi^{\prime}\right)-(\neg a)=\emptyset$, i.e. $\mathcal{M}\left(\psi^{\prime}\right)=\neg a$, which means that we can derive both $a$ and $\neg a$ from $s$, and hence $s$ is inconsistent.

Lemma 4.1. Procedure Saturate $(s)$ returns a SOK $s_{0}$ that is a saturated equivalent of $s$, if $s$ is consistent, or fail, otherwise. Assuming $s$ consists of $n$ fixed length $\psi$-forms and $m$ atoms, the time complexity of procedure Saturate(s) is $O\left(n m^{C}\right)$, where $C$ denotes the maximum length of a $\psi$-form clause.

Proof. The SOK returned by Saturate (Figure 12) contains the input set of propositions $s$ and some additional propositions that are derived from $s$ using unit clause resolution, i.e. those that follow from $s$. Thus the returned SOK $s_{0}$ is equivalent to the input.

It is saturated because we compute and add the results of all possible resolutions to $s_{0}$. Thus, for every ground proposition $p$ such that $s \models p$, there is a proposition $c \in s_{0}$ such that $c \models p$.

Saturate computes all possible resolutions in $s$ and returns fail whenever an empty disjunct is derived, as follows from the the known property of resolution deduction (see [20] page 87): If a set $\Delta$ of ground clauses is unsatisfiable, then there is a resolution deduction of the empty clause from $\Delta$.

To estimate the time complexity bound, we consider the following stages of the algorithm. During the first stage, for each of $\mathrm{n} \psi$-forms in $s$ the procedure will first compute the resolution with every atom in $s$, when the resolution rule is applicable. Determining if resolution between an atom and a single fixed length $\psi$-form is applicable consists of computing an image of a single literal onto a $\psi$-form (step 4 in Figure 12), which takes 
constant time, assuming the number of exceptions, clauses and variables in a $\psi$-form are constant bounded. Thus, the first stage takes time $O(m n)$.

Note that the result of each resolution is another $\psi$-form (denoted $\psi_{n e w}$ in the algorithm), which is added to the saturation $s_{0}$. For each $\psi$-form at most $m$ new $\psi$-forms can be added to $s_{0}$ as a result of resolution with the atoms in $s$. Furthermore, each of the added $\psi$-forms will have 1 fewer literals in the main clause than the original. Overall, $\mathrm{nm}$ new $\psi$-forms with the maximum clause length $C-1$ could be added to $s_{0}$ during the first stage.

During the second stage, resolutions are computed between the $\psi$-forms added in the previous stage and $m$ atoms of $s$. This process will take time $O\left(m^{2} n\right)$ and add no more than $m^{2} n$ new $\psi$-forms with the maximum clause length of $C-2$.

The third stage will take $O\left(m^{3} n\right)$ time and add no more than $m^{3} n$ new $\psi$-forms with the clause length $C-3$, and so on. Since the maximum possible length of the main clause in the $\psi$-forms added at each stage is decreasing by one at each stage of this process, it is evident that the number of stages is bounded by the size of the longest $\psi$-form clause $C$. Overall computation will thus take time $O\left(m n+m^{2} n+\ldots+m^{C} n\right)=O\left(m^{C} n\right)$.

When a set of PSIPLAN propositions $s$ consists of $m$ atoms and $n \psi$-forms, checking $s \models a$, where $a$ is an atom, takes time $O(m)$ because a set of PSIPLAN propositions only entails those atoms that it contains. Checking $s \models \psi$ takes time $O\left(n m^{C}+n\right)$, where $O\left(n m^{C}\right)$ is the time to saturate $s$. When $s$ is saturated, checking $s \models \psi$ is $O(n)$.

4.3. PSIPLAN Actions. Actions are deterministic and are represented via preconditions and effects. Actions are described using parameterized schemas, however, for the simplicity of presentation, the examples in this section present instantiated, i.e. fully grounded, versions of actions.

Each action $a$ has a name, $\mathcal{N}(a)$, and a set of preconditions, $\mathcal{P}(a)$, which identify the domain propositions necessary for executing the action. The propositions in $\mathcal{P}(a)$ can include literals and quantified $\psi$-forms ${ }^{4}$.

Each domain action has a set of domain literals called the assert list, $\mathcal{A}(a)$. The assert list, also called the effects of the domain action, identifies the complete set of domain propositions whose value may change as a result of the action. We assume that an action is deterministic and can change the truth value (true or false) of only a finite number of atoms, and thus any $\psi$-form in the assert list defines a single negated literal.

Consider, for example, PSIPLAN encoding of the action of moving a file from one directory to another, as depicted in Figure 13. $m v(F, S, D)$ moves file $F$ from directory $S$ into the directory $D$. The single precondition requires that file $F$ be in directory $S$. The effects are given by a $\psi$-form that denotes that file $F$ is not in directory $S$, and an atom that denotes that file $F$ is in directory $D$. Action $\operatorname{lift}(B, L)$, from our warehouse domain, lifts object $B$ from location $L$. One of its preconditions is a quantified $\psi$-form and requires that $B$ contains no fragile goods.

\footnotetext{
${ }^{4}$ Ruling out other forms of non-quantified disjunction is not a limitation, since any action schema with a non-quantified disjunction as its precondition can be equivalently split into several actions.
} 


$$
\begin{array}{cc}
\text { PSIPLAN action } a=m v(F, S, D) & \text { PSIPLAN action } a=\operatorname{lift}(B, L) \\
\mathcal{P}(a): \operatorname{In}(F, S), F i l e(F), \operatorname{Dir}(S), \operatorname{Dir}(D) & \mathcal{P}(a):[\neg \operatorname{In}(g, B) \vee \neg F r a g i l e(g)], \operatorname{At}(B, L) \\
\mathcal{A}(a):[\neg \operatorname{In}(F, S)], \operatorname{In}(F, D) & \mathcal{A}(a):[\neg A t(B, L)], \operatorname{Lifted}(B)
\end{array}
$$

Figure 13: PSIPLAN domain actions

4.4. Planning Problem. A planning problem is a three tuple $\langle\Lambda, \mathcal{I}, \mathcal{G}\rangle$ where $\Lambda$ is the set of available PSIPLAN actions, $\mathcal{I}$ is the set of initial conditions - i.e., a set of PSIPLAN propositions - and $\mathcal{G}$ is the goal, which is also a set of PSIPLAN propositions.

A solution plan is a sequence of actions, that is executable and transforms any world state satisfying the initial conditions into a world state satisfying the goal.

Given a sequence of ground actions $a_{1}, \ldots, a_{n}$, let $W_{i}$ denote the set of possible worlds obtained by executing the sequence up to the i-th action from any of the possible initial worlds. Let $W_{0}$ denote the set of possible worlds corresponding to the initial conditions, i.e. $I(\mathcal{I})$. Then, a sequence of actions $a_{1}, \ldots, a_{n}$ is called a solution plan to a planning problem $\langle\Lambda, \mathcal{I}, \mathcal{G}\rangle$, if and only if:

(1) The goal $\mathcal{G}$ holds in all final worlds, i.e. for all $w$ in $W_{n}, w(\mathcal{G})$, and

(2) Each action $a_{i}$ of the plan is executable in every possible world $w$ in $W_{i}$, for all values of $i, 0 \leq i<n$, i.e. for all $w$ in $W_{i}, w\left(\mathcal{P}\left(a_{i}\right)\right)$.

Since our agent uses the SOK $s$ to represent the set of possible worlds $I(s)$, in order to plan, it must be able to progress the SOK in order to predict the set of worlds resulting from executing a sequence of actions. The function update() does exactly that.

If $\vec{a}$ is a sequence of actions executable from the SOK $s_{0}$, update $\left(\vec{a}, s_{0}\right)$ denotes the SOK our agent uses to predict the set of possible worlds resulting from executing $\vec{a}$ in any of the worlds $I\left(s_{0}\right)$.

Ideally, the SOK obtained by progression must include all and only worlds that are the result of executing the sequence $\vec{a}$ in some world described by the initial SOK. Then, every plan that is executable and achieves the goal in the agent's knowledge of the world is indeed a solution plan for the real world. This requirement is satisfied when the update() function is correct and complete.

The next section formally defines the correctness and completeness properties, presents PSIPLAN's update procedure and proves that it is correct and complete. Thus, a sequence of ground actions $a_{1}, \ldots, a_{n}$, is a solution to the planning problem $\langle\Lambda, \mathcal{I}, \mathcal{G}\rangle$ if and only if

(1) The goal $\mathcal{G}$ is entailed by the final SOK, i.e. update $\left(a_{1} \ldots a_{n}, \mathcal{I}\right) \models \mathcal{G}$, and

(2) Each $a_{i}$ is executable, i.e. update $\left(a_{1} \ldots a_{i-1}, \mathcal{I}\right) \models \mathcal{P}\left(a_{i}\right)$.

Thus, goal achievement can be established by checking entailment from the updated SOK without considering the set of all possible worlds.

4.5. SOK Update. Actions cause transitions between worlds. The agent's SOK must evolve in parallel with the world, and must adequately reflect the changes in the world that occur due to an action. Correctness of a SOK update guarantees that the SOK is always consistent with the world model, given a consistent initial SOK. The other desirable property of the SOK update is completeness: we would like the agent to take advantage of 
all information that becomes available and not to discard what was previously known and has not changed. The correctness and completeness properties of the SOK update, as well as soundness and completeness of entailment within the state language, are prerequisites for a sound and complete planning algorithm. The correctness and completeness criteria are best formulated in the context of possible worlds. Let $d o(a, W)$ denote the set of worlds obtained from performing action $a$ in any of the worlds in $W$, and update $(s, a)$ denote the SOK that results if the agent performs action $a$ from SOK $s$. We say that the update procedure is correct if and only if

$$
I(\operatorname{update}(s, a)) \subseteq \operatorname{do}(a, I(s)),
$$

i.e. every possible world after performing the action $a$ has to have a possible predecessor.

The update procedure is complete if and only if

$$
d o(a, I(s)) \subseteq I(\text { update }(s, a)),
$$

i.e. every world obtained from a previously possible world is a model of the new SOK. This implies that all changes to the world must be reflected in the new SOK.

To achieve correctness of SOK updates, the agent must remove from the SOK all propositions whose truth value might have changed as the result of the performed action. To achieve completeness, the agent must add to the SOK all facts that become known. The complexity of the SOK update, therefore, depends critically on the process of identifying the propositions that must be retracted to preserve correctness. In our language, this computation is reduced to computing e-difference, which has polynomial complexity.

To obtain the agent's SOK $s$ after performing an action $a$, we first remove all propositions implied by the negation of the assert list, as only those propositions of $s$ might change their values after $a$. Symbol $\mathcal{A}^{-}(a)$ is used to denote the set of negations of propositions in the assert list of $a$. For example, the action $a=m v(f i g, / i m g, / t e x)$ of moving file $f i g$ from directory /img into /tex has assert list

$$
\mathcal{A}(a)=\{[\neg \operatorname{In}(\text { fig }, / \text { img })], \operatorname{In}(\text { fig }, / \text { tex })\},
$$

and thus

$$
\mathcal{A}^{-}(a)=\{\operatorname{In}(\text { fig }, / \text { img }),[\neg \operatorname{In}(\text { fig }, / \text { tex })]\} .
$$

During the update we also remove from the SOK all redundant propositions, i.e. those that follow from the effects of the action, and then add these effects to the new state. The agent's SOK after executing action $a$ in the SOK $s$ is described below.

$$
\text { update }(s, a)=\left(\left(s \underline{e} \mathcal{A}^{-}(a)\right) \underline{e} \mathcal{A}(a)\right) \cup \mathcal{A}(a)
$$

Though it is not necessary, we include $\underline{e} \mathcal{A}(a)$ in (4.4) to keep the SOK simple. $\underline{e} \mathcal{A}(a)$ removes all propositions entailed by $\mathcal{A}(a)$, not just those in $\mathcal{A}(a)$.

Example 4.2. For an example, consider action $a=m v$ (fig, /img, /tex) introduced above. Let $\mathcal{P}(a)=\{\operatorname{In}($ fig, $/$ img $)\}$, which states that fig must be in /img. Recall that $\mathcal{A}(a)=$ $\{[\neg \operatorname{In}($ fig,$/$ img $)], \operatorname{In}($ fig,$/$ tex $)\}$ and $\mathcal{A}^{-}(a)=\{\operatorname{In}($ fig, $/$ img $),[\neg \operatorname{In}($ fig, $/$ tex $)]\}$.

We begin with an SOK $s$, which states that fig and a.bmp are the only files in /img, and that a.ps is the only Postscript file in the system, except for possibly files in directory 
/img.

$$
s=\left\{\begin{array}{l}
\operatorname{In}(\text { fig }, / \text { img }), \operatorname{In}(\text { a.bmp }, / i m g), T(\text { a.ps }, P S), \\
{[\neg \operatorname{In}(x, / i m g) \text { except }\{\{x=\text { fig }\},\{x=a . b m p\}\}],} \\
{[\neg \operatorname{In}(x, d) \vee \neg T(x, P S) \text { except }\{\{x=a . p s\},\{d=/ i m g\}\}]}
\end{array}\right\}
$$

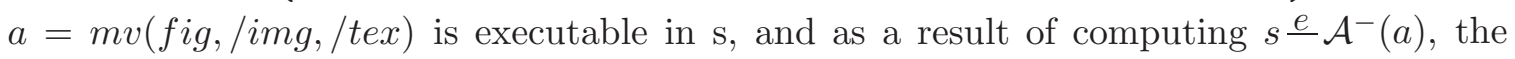
atom $\operatorname{In}(\mathrm{fig}, / \mathrm{img})$ is removed from $s$ and the exception $\{x=f i g, d=/$ tex $\}$ is added to the second $\psi$-form. The first $\psi$-form is left intact, producing

$$
s \underline{e} \mathcal{A}^{-}(a)=\left\{\begin{array}{l}
\operatorname{In}(\text { a.bmp, } / \text { img }), T(\text { a.ps }, P S), \\
{[\neg \operatorname{In}(x, / \text { img }) \text { except }\{\{x=\text { fig }\},\{x=\text { a.bmp }\}\}],} \\
{[\neg \operatorname{In}(x, d) \vee \neg T(x, P S) \text { except }} \\
\{\{x=\text { a.ps }\},\{d=/ \text { img }\},\{x=\text { fig }, d=/ \text { tex }\}\}]
\end{array}\right\} .
$$

Further e-difference with $\mathcal{A}(a)$ and union with $\mathcal{A}(a)$ yields the following SOK

$$
s^{\prime}=\left\{\begin{array}{l}
\operatorname{In}(\text { fig }, / \text { tex }), \operatorname{In}(\text { a.bmp, } / \text { img }), T(\text { a.ps }, P S), \\
{[\neg \operatorname{In}(x, / \text { img }) \text { except }\{\{x=\text { fig }\},\{x=\text { a.bmp }\}\}],} \\
{[\neg \operatorname{In}(x, d) \vee \neg T(x, P S) \text { except }\{\{x=\text { a.ps }\},\{d=/ i m g\},\{x=f i g, d=/ \text { tex }\}\}]} \\
{[\neg \operatorname{In}(\text { fig }, / \text { img })]}
\end{array}\right\} .
$$

Note that $s$ contained $\neg \operatorname{In}($ fig, /tex $) \vee \neg T($ fig, $P S)$ and that we added $\operatorname{In}($ fig, /tex $)$ when determining $s^{\prime}$. If our update rule retained $\neg \operatorname{In}($ fig, /tex $) \vee \neg T($ fig, PS $)$ in $s^{\prime}$, then in $s^{\prime}$ we could perform resolution and conclude that $\neg T($ fig, $P S)$. However, this would be wrong because we have no information on whether or not fig is a Postscript file. Instead, our update rule removes any clause that is entailed by $\neg \operatorname{In}($ fig,/tex $)$, and so $s^{\prime}$ does not contain $\neg \operatorname{In}($ fig,$/$ tex $) \vee \neg T($ fig, $P S)$.

This update rule (4.4) produces the same result as Winslett's update operator [41] in the special case where actions are deterministic. Moreover, our rule accomplishes this without considering all possible worlds corresponding to SOK $s$ explicitly, and thus is more efficient.

We show next how the same rule is used for updating the state of knowledge after the actions that create new objects ${ }^{5}$.

Example 4.3. Consider an action of creating a new file named afile in directory / code with effect In (afile,/code) and no preconditions. When this action is executed in $s^{\prime}$, the state update rule yields the new SOK $s^{\prime \prime}$ that differs from $s^{\prime}$ in the following way:

(1) $s^{\prime \prime}$ contains a new atom In (afile,/code), reflecting the effect of the action added by the update rule,

(2) the first $\psi$-form of $s^{\prime}$ now has a new exception, reflecting the fact that it is unknown whether afile has Postscript format. This is the result of the e-difference $s^{\prime} \stackrel{e}{\{}\{\neg \operatorname{In}($ afile,$/$ code $\left.)]\right\}$.

\footnotetext{
${ }^{5}$ Note that discovering a new object is a different issue (since it assumes that the object always existed in the domain and is therefore included in the universal quantification even prior to being discovered, which is not true of a newly created object) that is also handled in PSIPLAN, however it is beyond the scope of this paper.
} 


$$
s^{\prime \prime}=\left\{\begin{array}{l}
\text { In (afile, } / \text { code }), \operatorname{In}(\text { fig }, / \text { tex }), \operatorname{In}(\text { a.bmp }, / \text { img }), T(\text { a.ps }, P S), \\
{[\neg \operatorname{In}(x, d) \vee \neg T(x, P S) \text { except }\{\{x=\text { a.ps }\},\{d=/ \text { img }\},\{x=\text { fig }, d=/ \text { tex }\},} \\
\{x=\text { afile, } d=/ \text { code }\}\}] \\
{[\neg \operatorname{In}(x, / \text { img }) \text { except }\{\{x=\text { fig }\},\{x=\text { a.bmp }\}\}],} \\
{[\neg \operatorname{In}(\text { fig }, / \text { img })]}
\end{array}\right\}
$$

A factor that turns out to be critical for the use of PSIPLAN in planning is that the SOK resulting from updating a saturated SOK is also saturated. After the initial SOK of the agent is saturated, there is no need to consider resolution of the initial conditions and action effects in satisfying a goal.

We call an SOK $s$ minimal if it is saturated and it does not contain any ground clause entailed by some other clause in $s$, i.e. for any two ground clauses $p, q$ from $s$

$$
(q \models p) \Longrightarrow(q=p)
$$

Theorem 4.4. Let a be a domain action and $s$ be an agent's SOK before executing a, where $s$ is satisfiable and saturated, and $a$ is executable in $s$. Then the following state of knowledge update rule

$$
\text { update }(s, a)=\left(\left(s \stackrel{e}{-} \mathcal{A}^{-}(a)\right) \stackrel{e}{-} \mathcal{A}(a)\right) \cup \mathcal{A}(a),
$$

is correct and complete. Moreover, the resulting $S O K s^{\prime}=$ update $(s, a)$ is saturated. It is minimal if $s$ is minimal.

Proof. We start by proving that the result of updating a saturated SOK is a saturated SOK. We first show that if $s$ is saturated then $s_{1}=\left(\left(s \underline{e} \mathcal{A}^{-}(a)\right) \underline{e} \mathcal{A}(a)\right)$ is also saturated ( recall that $\mathcal{A}^{-}(\mathbf{a})$ is used to denote the set of negations of propositions in the assert list of $a$ ). Suppose this is not true, i.e. there's a ground PSIPLAN proposition $p$ such that, while $s_{1} \models p, \forall p^{\prime} \in s_{1} \cdot p^{\prime} \not \forall p$. Let $q$ be a smallest (non-empty) subclause of $p$ such that $s_{1} \models q$ (there might be several such $q$ ). Since $s_{1} \subseteq s, q$ must also be entailed by $s$, but $s$ is saturated, so there exists a $q^{\prime}$ in $s$ such that $q^{\prime} \models q$, i.e. $q^{\prime} \subseteq q$.

$q^{\prime} \notin s_{1}$ (otherwise $q^{\prime}$ as a subclause of $p$ would entail $p$ ) so $q^{\prime}$ must be entailed by either $\mathcal{A}(a)$ or $\mathcal{A}^{-}(a)$. We abbreviate the union $\mathcal{A}(a) \cup \mathcal{A}^{-}(a)$ by $\mathcal{A}^{\circ}(a)$. Since $q^{\prime} \in(\mathcal{A}(a) \triangleright s) \cup$ $\left(\mathcal{A}^{-}(a) \triangleright s\right)$, there must be a literal $e$ in $\mathcal{A}^{\circ}(a)$ such that $e$ is a subclause of $q^{\prime}$, and therefore, $e$ is a subclause of $q$. Note, that since $s_{1} \models q$, in case $q \notin s_{1}$ there must be a way of deriving $q$ by resolution from some propositions of $s_{1}$, which is only possible when there exists a proposition $r$ in $s_{1}$ such that $r$ contains $q$ as a subclause. This means that $r$ has $e$ as a subclause, and consequently $r$ is entailed by $e$. But according to the definition of $s_{1}$, it does not contain any clauses entailed by any clause in $\mathcal{A}^{\circ}(a)$. We arrive at a contradiction, so $s_{1}$ is saturated.

Adding $\mathcal{A}(a)$, which consists of literals and is itself saturated, to $s_{1}$ also produces a saturated state. Assume there is a proposition $q$ that is not entailed by either $s_{1}$ or some element of $\mathcal{A}(a)$, but is entailed by $s_{1} \cup \mathcal{A}(a)$. $q$ cannot be an atom, therefore it is a negated clause. The only clauses that are not entailed by $\mathcal{A}(a)$ and $s_{1}$ separately, but are entailed by their union are those obtained via resolution of some atom $e \in \mathcal{A}(a)$ with a ground clause of the form $\neg e \vee x$ in $s_{1}$. But this is impossible, because $\mathcal{A}^{-}(a) \models \neg e \vee x$, so such clause would not be in $s_{1}$. We arrive at a contradiction.

In case $s$ was minimal, $s^{\prime}$ is also minimal, because

- removing clauses from a minimal set preserves minimality, so $s_{1}$ is minimal, and - $s^{\prime}=s_{1} \cup \mathcal{A}(a)$ is minimal because

(1) $s_{1}$ does not contain any propositions entailed by any literal in $\mathcal{A}^{\circ}(a)$, 
(2) $\mathcal{A}(a)$ is minimal, and

(3) $s_{1}$ does not entail anything in $\mathcal{A}(a)$.

To prove that the update function is correct and complete, we need to show that for $s^{\prime}=\operatorname{update}(s, a)$ :

$$
I\left(s^{\prime}\right)=d o(a, I(s)) .
$$

Completeness proof. We first show that $d o(a, I(s)) \subseteq I\left(s^{\prime}\right)$, i.e. for every world $w$ in $I(s)$, its successor, $w^{\prime}=d o(a, w)$, is in $I\left(s^{\prime}\right)$. The set of possible worlds consists of all and only worlds that model the agent's knowledge of the world, i.e. for any $s I(s)=$ $\{w \mid(p \in s) \Longrightarrow w(p)=$ true $\}$. Thus, to show that $w^{\prime} \in I\left(s^{\prime}\right)$ we need to prove, that for every proposition $p$ in $s^{\prime}, w^{\prime}(p)=$ true.

Note that according to the world transition model, $w^{\prime}=\left(w-\mathcal{A}^{\circ}(a)\right) \cup \mathcal{A}(a)$.

We partition $s^{\prime}$ into $s_{1}^{\prime}=\left(\left(s \underline{e} \mathcal{A}^{-}(a)\right) \underline{e} \mathcal{A}(a)\right)$ and $s_{2}^{\prime}=\mathcal{A}(a)$. We partition $w^{\prime}$ into $w_{1}^{\prime}=w-\mathcal{A}^{-}(a)-\mathcal{A}(a)$ and $w_{2}^{\prime}=\mathcal{A}(a)$. Since $w \in I(s)$, for every $p_{1}$ such that $p_{1} \in s_{1}^{\prime}$, $w_{1}^{\prime}\left(p_{1}\right)$. Also, for each $p_{2} \in s_{2}^{\prime}$, we have $w_{2}^{\prime}\left(p_{2}\right)$. Therefore for every $p$ such that $p \in s, w(p)$.

Correctness proof. Now we need to show that $I\left(s^{\prime}\right) \subseteq d o(a, I(s))$, i.e. every possible world $w^{\prime}$ of $s^{\prime}$ has a predecessor $w$ that is a possible world of $s$. We need to show that for every $w^{\prime} \in I\left(s^{\prime}\right)$ there is a world $w$ such that $w^{\prime}=\left(w-\mathcal{A}^{\circ}(a)\right) \cup \mathcal{A}(a)$ where $w$ is in $I(s)$ and $\mathcal{A}^{\circ}(a)$ denotes the union $\mathcal{A}(a) \cup \mathcal{A}^{-}(a)$. A possible world is a model of all propositions $p$ such that $p \in s$, i.e. $w$ is in $I(s)$ if and only if $w$ models every such domain proposition $p$.

The proof is by construction. Since $s$ is saturated, for every proposition $p$ that is implied by $s$, there's a single proposition $q \in s$ such that $q \models p$.

STEP 1. Since $w^{\prime}=d o(a, w)$ we need to include in $w$ all literals of $w^{\prime}$ that are not in $\mathcal{A}(a)$, because those would not have changed as a result of the action. Let $w_{0}=w^{\prime}-\mathcal{A}(a)$ and $w$ will include $w_{0}$.

STEP 2. We also include in $w$ those literals from $\mathcal{A}(a)^{\circ}$ that are known in $s$, i.e. the literals $l \in \mathcal{A}^{\circ}(a)$ such that $l \in s$.

STEP 3. At this point every literal or its complement is included in $w$ except for $l \in \mathcal{A}^{\circ}(a)$ where neither $l$ nor $\neg l$ belongs to $s$. We now describe a procedure for choosing either the literal or its complement for inclusion in $w$ from these "leftover" literals. Suppose $\neg l$ is an arbitrary negated literal from this set. Further, let $C=\neg l \triangleright s$. If there is a proposition in $C$ that is not already implied by some $p$, where $p \in w$, then we must include $\neg l$ in $w$ in order to keep it accessible from $s$. Otherwise we may include in $w$ either $l$ or $\neg l$.

The world $w$ is now completely specified and is easy to verify that $w \in I(s)$, as well as $w^{\prime}=d o(a, w)$.

We should note that although the state update procedure above is defined for fully grounded actions, it does not mean that all PSIPLAN-based planners must work with fully grounded representations. For example, the partial order planner PSIPOP operates using action schemas and grounds action parameters only as needed.

\section{Related Work}

5.1. Representations for conformant planning. Representations used for reasoning and planning in an open world can be broadly categorized as those that operate using the set of all possible worlds, and those that rely instead on reasoning using a specification of incomplete state of knowledge. Presented here PSIPLAN belongs to the second of these categories. 
Among the planning systems in the second category is a situation-calculus based planner by Finzi et al. [18, implemented in GOLOG [27]. The planning task is reduced to theorem proving in situtation calculus, and the authors present two approaches to theorem proving from the initial state. One approach invokes a Davis-Putnam based theorem prover every time entailment from initial situation is checked. The other approach intends to minimize the time spent checking entailment by precompiling the specification of the initial state into its equivalent form containing all prime implicates of the original specification. (The reduction to prime implicates is akin to PSIPLAN's saturation of the initial state.) From the prime-implicate form further theorem proving is done by subsumption of clauses.

The foregoing is the only conformant planner that subsumes the state and goal language of PSIPLAN. However, the examples presented in the paper do not contain universally quantified disjunctive goals with exceptions that are handled by PSIPLAN. The generality of the situation-calculus permits any first-order specification of the initial and goal situations, and actions, however, at a price of the complexity of planning. In PSIPLAN, we have deliberately and significantly restricted the language for the sake of reduced complexity of reasoning.

A subset of situation calculus with equality, which has tractable, sound and under certain conditions complete action progression procedure from an incompletely specified state is presented by Liu and Levesque in [30. There are similarities between PSIPLAN and the language of Liu and Levesque, in particular in the use of universally quantified statements in the knowledge base. However, neither language subsumes the other one in the expressive power.

Shirazi and Amir ([35) also address the problem of progressing a belief state encoded in first-order logical sentences over a sequence of actions. They present special purpose algorithms for computing the progression, which they call logical filtering, in polynomial time. The polynomial time complexity of belief update is achieved for STRIPS and also permuting actions. An action is called permuting, if for each world $w^{\prime}$ there is at most one $w$ such that $d o(a, w)=w^{\prime}$, i.e. for every world potentially resulting from execution of action $a$, there is a unique "original" world state. PSIPLAN's actions are not permuting, however they are similar to STRIPS actions in the sense that the assert list of an action includes only those literals that change as the result of an action and there are no conditional effects. Thus, the polynomial time complexity of PSIPLAN's update procedure is consistent with the findings of Shirazi and Amir.

Eiter et al. [14, 15] propose a (propositional) logic based planning language $\mathcal{K}$ for planning with incomplete information as answer set programming. In this framework, proposed originally by Lifschitz [29], a plan is the answer set of a logical program formulated using a specialized logical language. $\mathcal{K}$ represents lack of knowledge using negation as failure semantics. It supports both knowledge state and possible world planning. The authors further distinguish between optimistic and secure (i.e. conformant) planning. Optimistic plans may not be executable, due to their assumptions on the missing information. $\mathcal{K}$ supports conditional effects, but does not allow any universal quantification on goals or state description.

Thielscher [37] presents FLUX - a logical programming framework for agent program design in the presence of incomplete information and sensing. FLUX is based on fluent calculus and is implemented as a set of constraints, defining the domain, action update, agent's knowledge and action execution. The syntax of the language is carefully restricted 
to provide linear time evaluation of the constraints. The constraint language includes universally quantified negated clauses, similar to the simple $\psi$-forms of PSIPLAN. However, unlike PSIPLAN, the constraint solver assumes a finite domain, and does not represent exceptions to the universally quantified clauses. FLUX has nice computational properties, but it is not complete. Also differently from PSIPLAN, the FLUX framework is designed for programming the intended behavior of the agent via a designer-specified strategy, which defines the set of agent control rules, rather than the problem of automatically constructing a sequence of actions that will result in the achievement of the goal.

Conformant Graphplan [36] and its extension to planning with sensing, SGP [40] are propositional Graphplan [9] based open world planners that consider every possible world and thus rely on the domain of objects being sufficiently small. However, in small domains these planners are able of generating remarkably long plans. Graphplan based planners perform a search in a space of graphs generated by forward-chaining in the state space, and their performance degrades when the initial state contains large number of irrelevant atoms.

CMBP planner [13] is a conformant planner based on model checking. Like Conformant Graphplan it performs a forward-chaining analysis, but relies on an effective way of encoding sets of possible worlds and its performance is less dependent on the amount of irrelevant information in the initial state. CMBP uses action representation in the form of nondeterministic state transition relations.

An approach to conformant planning as a heuristic search in the space of belief states that are sets of world states is presented by Bonet and Geffner ([10]). An admissible heuristic function is computed based on the distance to the goal state under the assumption of complete information. The search produces an optimal plan, however the algorithm relies on the finiteness of the state space, which is not achievable when the domain of objects is infinite. The action language used is an extension of STRIPS that includes function symbols, negation, disjunction, non-deterministic actions and conditional effects.

IPE [1, SENSE-P [17, XII 22, PUCCINI 21] are causal link planners that interleave planning with execution of incomplete plans. The action description language of PUCCINI, SADL [23] includes actions with conditional and informational effects. However, to the best of our knowledge there are no completeness results for conformant planning with SADL.

PKS 33] is a forward chaining planner based on a representation of the agent's knowledge that captures a set of possible worlds via a set of knowledge formulas similarly to PSIPLAN's SOK. The representation of Petrick and Baccus includes functional symbols, conditional plans and actions with conditional effects, all of which are not represented in PSIPLAN for the reason of tractability. However, the PKS planner only admits ground literals in its goal language and does not handle universally quantified negated goals. PKS is also incomplete, but the authors report that it is able to generate plans in many domains.

The LCW [16] (see introduction) language is extended in [28, 19] to handling exceptions. Levy in 28] uses extended LCW sentences, called Local Completeness (LC) sentences, to represent database completeness information and derive answer completeness property of a conjunctive query. This is analogous to computing whether a SOK entails a universally quantified knowledge goal, where the SOK is given by the combination of relational tables and the knowledge goal is to know all individual objects that satisfy a given query. Friedman and Weld [19] extend on Levy's work for the purpose of eliminating redundant information gathering from databases by an Internet agent. They present a method of determining subsumption from LC sentences: whether a set of relational tables contains all information 
available in another relational table. Thus, both of these works only consider the setting in which there are no actions that can change the world, do not address a changing world or planning, and do not present any methods that would make these extensions amenable to their use in an open world planning algorithm, such as the image and e-difference operations of PSIPLAN that are critical in the computation of state update after an action, causal links and threat resolution.

5.2. Complexity. Complexity of propositional planning with incomplete information, with and without sensing actions, has been addressed by many researchers (e.g. [24, 15], 6], [38]). Results presented in these works, although for different state and action languages, generally show that the complexity of constructing conformant plans of polynomial length is greater than planning with complete information, which is NP-complete. Though we have not proven the following formally, from the results of this paper it appears that: (a) checking whether a given plan (of polynomial length) solves a given problem in PSIPLAN can be done in polynomial time and, thus, (b) determining whether there exists a plan (of polynomial length) that solves a given problem in PSIPLAN is NP-complete. These results do not contradict the results of Baral et al., nor those of Turner [38, as we explain below; the key to the reduced complexity of PSIPLAN-based planning compared to the analysis in these papers seems to be the absence of conditional effects.

Baral et al. 6] present complexity results for a variety of problems related to open world planning with action language $\mathcal{A}$. In particular, they show the problem of finding all solution plans in presence of incomplete information and no sensing belongs to the class $\Sigma_{2} P$. To keep the complexity of planning with incomplete information within the NP-completeness bounds, they propose a 0-approximation, which sacrifices completeness.

In PSIPLAN as in 0-approximation of Baral et al., the set of possible worlds is represented by a set of propositions that are known to be true. However, unlike the action language A used in Baral et al.'s work, PSIPLAN's action language does not allow for conditional effects, and so all of an action's effects are guaranteed to be true after the (executable) action is performed. In contrast, in action language A determining the effect of the action and thus the resulting set of possible worlds sometimes requires an analysis of possible values of unknown propositions. 0-approximation does not involve such analysis and thus loses such plans.

For example having no information at all and an action $a_{0}$ with conditional effect " $a_{0}$ causes $\mathrm{p}$ if $\neg p$ ", a plan that consists of a single action $a_{0}$ achieves $p$, but it will be missed by 0 -approximation. Without the analysis of the result of performing $a_{0}$ in two possible initial states (corresponding to the two different values of $\mathrm{p}$ ), it is impossible to conclude that $p$ is true after performing $a_{0}$. That is the reason why 0 -approximation will miss it. In PSIPLAN, there are no conditional effects, and so action $a_{0}$ from above cannot be represented. Once executability of an action is determined, all effects are guaranteed and the set of possible worlds is precisely described by the single updated state of knowledge. Thus, in PSIPLAN completeness of conformant planning is preserved without an increase of complexity over classical planning.

Turner 38 presents a comprehensive complexity analysis of a set of planning problems by using a very general framework for describing a planning problem. This framework represents actions as state transition relations and integrates many action languages including those with conditional effects, nondeterminism and concurrency. As in Baral et al. his 
results on conformant planning consider actions that may have conditional effects, and are more general than PSIPLAN's.

Haslum and Jonsson's paper 24] states a PSPACE-completeness result for the problem of verifying existence of a conformant plan of unbounded length with STRIPS-like actions. This result is presented without proof and thus it is difficult to analyze it for the case of polynomially bounded-length plans.

\section{Conclusions}

Classical planning presupposes that complete and correct information about the world is available at any point of planning (by having a completely specified initial situation, and deterministic actions). However, in a more realistic setting, the knowledge about the initial state may be incomplete, the effects of actions may be nondeterministic, or there may be other agents acting in the world. These are some sources of uncertainty in planning.

In this paper we dropped one of the assumptions of classical planning - the assumption of complete knowledge of the initial state of the world - thereby considering the problem of open world planning. We have presented PSIPLAN, a language for representing and reasoning in open world applications. PSIPLAN uses $\psi$-forms to represent infinite sets of clauses of negated literals. We have shown the following.

- Our algorithm to determine entailment in PSIPLAN is sound and complete and has polynomial complexity in the number of propositions in the state of knowledge under certain assumptions on the structure of $\psi$-forms common for open world planning problems. Operations image and e-difference between $\psi$-forms, which are crucial to planning with quantified propositions, also have polynomial complexity.

- Updating the agent's state of knowledge after performing an action has polynomial complexity in the number of propositions in the state of the agent's knowledge. In addition, the update procedure correctly and completely describes the transition between possible worlds due to the action.

Thus, PSIPLAN representation efficiently handles domains with an incomplete specification of the initial state without considering the set of all possible worlds, and does not require that the agent know the set of all objects. We implemented a partial order planning algorithm PSIPOP [5] for open worlds that uses PSIPLAN representation of state and actions. PSIPOP uses PSIPLAN calculus for reasoning about goal achievement. Since all of the PSIPLAN operations used by PSIPOP have only polynomial complexity, we argue informally that planning with PSIPLAN does not exceed the complexity of closed world STRIPS style planning. PSIGraph 12 is a GraphPlan-based planning algorithm which uses PSIPLAN.

Further evidence of the applicability of PSIPLAN representation for planning in open worlds with a large or infinite number of objects, is the use of PSIPOP's extension to planning with sensing and interleaved execution at the core of the Writer's Aid [3] - a collaborative bibliography assistant. Completeness and tractability of PSIPLAN's reasoning and its ability to effectively handle initial information and goal statements universally quantified over an infinite domain of objects ensured effective and non-redundant operation of the system, which were critically important in this application.

Experimental results from the initial implementation of PSIPOP, as well as an experimental assessment of the impact of various parameters of $\psi$-forms on the performance of the entailment algorithm are presented in [4. Results from the initial implementation 
of PSIGraph planner are presented in [12. We are currently working on optimizing the performance of these two planners. A thorough experimental evaluation of PSIPOP and PSIGraph is under way and we are planning on reporting it in a future paper.

In the future, we will extend PSIPLAN to allow function symbols, and we will publish already completed work that extends PSIPLAN to reasoning about the agent's knowledge goals and the effects of sensing actions.

\section{ACKNOWLEDGEMENTS}

We thank Barbara Grosz, Wendy Lucas, Wheeler Ruml and the anonymous referees for their helpful comments on an earlier draft of this paper.

\section{REFERENCES}

[1] Ambros-Ingerson, J. A. and S. Steel: 1988, 'Integrating Planning, Execution and Monitoring'. In: Proceedings of the Seventh National Conference on Artificial Intelligence (AAAI-88). St. Paul, Minnesota, pp. 83-88.

[2] Babaian, T.: 2000, 'Knowledge Representation and Open World Planning Using $\psi$-forms'. Ph.D. thesis, Tufts University, Medford, MA.

[3] Babaian, T., B. J. Grosz, and S. M. Shieber: 2002, 'A Writer's Collablrative Assistant'. In: Proceedings of Intelligent User Interfaces'02. pp. 7-14.

[4] Babaian, T. and J. Schmolze, 'Efficient Open World Reasoning and Planning'. Unpublished paper, available at http://cis.bentley.edy/tbabaian/papers/p1.ps.

[5] Babaian, T. and J. Schmolze: 2000, 'PSIPLAN: open world planning with $\psi$-forms'. In: Artificial Intelligence Planning and Scheduling: Proceedings of the Fifth International Conference (AIPS'00). pp. 292-300.

[6] Baral, C., V. Kreinovich, and R. Trejo: 2000, 'Computational complexity of planning and approximate planning in the presence of incompleteness'. Artificial Intelligence 122(1-2), 241-267.

[7] Baral, C. and T. C. Son: 1997, 'Approximate Reasoning about Actions in Presence of Sensing and Incomplete Information'. In: International Logic Programming Symposium. pp. 387-401.

[8] Biundo, S. and M. Fox (eds.): 1999, 'Proceedings of the 5-th European Conference on Planning'. Durham, England:, Springer-Verlag.

[9] Blum, A. L. and M. L. Furst: 1995, 'Fast Planning Through Planning Graph Analysis'. In: Proceedings of the Fourteenth International Joint Conference on Artificial Intelligence (IJCAI-95). Nagoya, Japan, pp. 1636-1642.

[10] Bonet, B. and H. Geffner: 2000, 'Planning with incomplete information as a heuristic search in belief space.'. In: Artificial Intelligence Planning and Scheduling: Proceedings of the Fifth International Conference (AIPS'00). Breckenridge, Colorado.

[11] Brafman, R. I. and J. Hoffmann: 2004, 'Conformant Planning via Heuristic Forward Search: A New Approach.'. In: ICAPS. pp. 355-364.

[12] Carlin, A., J. G. Schmolze, and T. Babaian: 2005, 'Graphplan Based Conformant Planning with Limited Quantification'. Research on Computing Science. 5 (Special Issue: Advances in Artificial Intelligence Theory), 65-75.

[13] Cimatti, A. and M. Roveri: 1999, 'Conformant Planning via Model Checking'. in [8].

[14] Eiter, T., W. Faber, N. Leone, G. Pfeifer, and A. Polleres: 2000, 'Planning under Incomplete Knowledge'. In: Computational Logic. pp. 807-821.

[15] Eiter, T., W. Faber, N. Leone, G. Pfeifer, and A. Polleres: 2004, 'A logic programming approach to knowledge-state planning: Semantics and complexity'. ACM Trans. Comput. Logic 5(2), 206-263.

[16] Etzioni, O., K. Golden, and D. Weld: 1997, 'Sound and efficient closed-world reasoning for planning'. Artificial Intelligence 89(1-2), 113-148.

[17] Etzioni, O., S. Hanks, D. Weld, D. Draper, N. Lesh, and M. Williamson: 1992, 'An Approach to Planning with Incomplete Information'. In: Proceedings of the Third International Conference on Principles of Knowledge Representation and Reasoning (KR-92). Cambridge, MA, pp. 115-125. 
[18] Finzi, A., F. Pirri, and R. Reiter: 2000, 'Open World Planning in the Situation Calculus'. In: Proceedings of the Seventeenth National Conference on Artificial Intelligence (AAAI-00). Austin, Texas.

[19] Friedman, M. and D. S. Weld: 1997, 'Efficiently Executing Information-Gathering Plans'. In: Proceedings of the Fifthteenth International Joint Conference on Artificial Intelligence (IJCAI-97).

[20] Genesereth, M. R. and N. Nilsson: 1986, Logical Foundations of Artificial Intelligence. MorganKaufmann Publishers.

[21] Golden, K.: 1997, 'Planning and knowledge representation for Softbots.'. Ph.D. thesis, University of Washington.

[22] Golden, K., O. Etzioni, and D. Weld: 1994, 'Omnipotence Without Omniscience: Efficient Sensor Management for Planning'. In: Proceedings of AAAI-94.

[23] Golden, K. and D. Weld: 1996, 'Representing Sensing Actions: The Middle Ground Revisited'. In: Proceedings of the Fifth International Conference on Principles of Knowledge Representation and Reasoning (KR-96). Cambridge, MA, pp. 174-185.

[24] Haslum, P. and P. Jonsson: 1999, 'Some results on the complexity of planning with incomplete information.'. in [8.

[25] Krebsbach, K., D. Olawsky, and M. Gini: 1992, 'An Empiracal Study of Sensing and Defaulting in Planning'. In: Artificial Intelligence Planning Systems: Proceedings of the First International Conference (AIPS-92). College Park, MD, pp. 136-144.

[26] Levesque, H.: 1996, 'What is planning in the presence of sensing?'. In: Proceedings of the Thirteenth National Conference on Artificial Intelligence (AAAI-96).

[27] Levesque, H. J., R. Reiter, Y. Lesperance, F. Lin, and R. B. Scherl: 1997, 'GOLOG: A Logic Programming Language for Dynamic Domains'. Journal of Logic Programming 31(1-3), 59-83.

[28] Levy, A.: 1996, 'Obtaining Complete Answers from Incomplete Databases'. In: Proceedings of the 22nd VLDB Conference. Mumbai (Bombay), India.

[29] Lifschitz, V.: 1999, 'Answer Set Planning.' In: ICLP. pp. 23-37.

[30] Liu, Y. and H. J. Levesque: 2005, 'Tractable reasoning with incomplete first-order knowledge in dynamic systems with context-dependent actions'. In: IJCAI. pp. 639-644.

[31] McAllester, D. and D. Rosenblitt: 1991, 'Systematic Nonlinear Planning'. In: Proceedings of the Ninth National Conference on Artificial Intelligence (AAAI-91). Anaheim, California, pp. 634-639.

[32] Peot, M. A. and D. E. Smith: 1992, 'Conditional Nonlinear Planning'. In: Artificial Intelligence Planning Systems: Proceedings of the First International Conference (AIPS-92). College Park, MD, pp. 189-197.

[33] Petrick, R. and F. Bacchus: 2002, 'A Knowledge-Based Approach to Planning with Incomplete Information and Sensing'. In: AI Planning and Scheduling (AIPS2002). pp. 212-222.

[34] Scherl, R. B. and H. J. Levesque: 1993, 'The Frame Problem and Knowledge-Producing Actions'. In: Proceedings of the Eleventh National Conference on Artificial Intelligence (AAAI-93).

[35] Shirazi, A. and E. Amir: 2005, 'First Order Logical Filtering'. In: IJCAI. pp. 589-595.

[36] Smith, D. and D. S. Weld: 1998, 'Conformant Graphplan'. In: Proceedings of the Fifteenth National Conference on Artificial Intelligence (AAAI-98).

[37] Thielscher, M.: 2005, 'FLUX: A logic programming method for reasoning agents'. Theory and Practice of Logic Programming 5(4-5), 533-565.

[38] Turner, H.: 2002, 'Polynomial-length planning spans the polynomial hierarchy'. In: Proceedings of Eighth European Conf. on Logics in Artificial Intelligence (JELIA'O2).

[39] Weld, D. S.: 1999, 'Recent Advances in AI Planning'. AI Magazine 20(2), 93-123.

[40] Weld, D. S., C. R. Anderson, and D. E. Smith: 1998, 'Extending Graphplan to Handle Uncertainty and Sensing Actions'. In: Proceedings of the Fifteenth National Conference on Artificial Intelligence (AAAI-98). Madison, Wisconsin.

[41] Winslett, M.: 1988, 'Reasoning about action using a possible models approach'. In: Proceedings of the Seventh National Conference on Artificial Intelligence (AAAI-88).

This work is licensed under the Creative Commons Attribution-NoDerivs License. To view a copy of this license, visit http://creativecommons.org/licenses/by-nd/2.0/ or send a letter to Creative Commons, 559 Nathan Abbott Way, Stanford, California 94305, USA. 